Mon. Not. R. Astron. Soc. 000, 000-000 (0000) Printed 9 December $2020 \quad$ (MN LATEX style file v2.2)

\title{
The Massive M31 Cluster G1: Detailed Chemical Abundances from Integrated Light Spectroscopy*
}

\author{
Charli M. Sakari ${ }^{1} \dagger$, Matthew D. Shetrone ${ }^{2,3}$, Andrew McWilliam ${ }^{4}$, George Wallerstein ${ }^{5}$ \\ 1 San Francisco State University, 1600 Holloway Avenue, San Francisco, CA, 94132 USA \\ 2 University of California Observatories/Lick Observatory, University of California, Santa Cruz, CA, 95064, USA \\ 3 McDonald Observatory, University of Texas at Austin, HC75 Box 1337-MCD, Fort Davis, TX 79734, USA \\ 4 Observatories of the Carnegie Institute of Washington, Pasadena, CA, USA \\ ${ }^{5}$ Department of Astronomy, University of Washington, Seattle WA 98195-1580, USA
}

9 December 2020

\begin{abstract}
G1, also known as Mayall II, is one of the most massive star clusters in M31. Its mass, ellipticity, and location in the outer halo make it a compelling candidate for a former nuclear star cluster. This paper presents an integrated light abundance analysis of $\mathrm{G} 1$, based on a moderately high-resolution $(R=15,000)$ spectrum obtained with the High Resolution Spectrograph on the Hobby-Eberly Telescope in 2007 and 2008. To independently determine the metallicity, a moderate resolution $(R \sim 4,000)$ spectrum of the calcium-II triplet lines in the near-infrared was also obtained with the Astrophysical Research Consortium's 3.5-m telescope at Apache Point Observatory. From the high-resolution spectrum, G1 is found to be a moderately metal-poor cluster, with $[\mathrm{Fe} / \mathrm{H}]=-0.98 \pm 0.05$. G1 also shows signs of $\alpha$-enhancement (based on $\mathrm{Mg}, \mathrm{Ca}$, and $\mathrm{Ti}$ ) and lacks the $s$-process enhancements seen in dwarf galaxies (based on comparisons of $\mathrm{Y}, \mathrm{Ba}$, and $\mathrm{Eu}$ ), indicating that it originated in a fairly massive galaxy. Intriguingly, G1 also exhibits signs of $\mathrm{Na}$ and $\mathrm{Al}$ enhancement, a unique signature of GCs - this suggests that G1's formation is intimately connected with GC formation. G1's high $[\mathrm{Na} / \mathrm{Fe}]$ also extends previous trends with cluster velocity dispersion to an even higher mass regime, implying that higher mass clusters are more able to retain Na-enhanced ejecta. The effects of intracluster abundance spreads are discussed in a subsequent paper. Ultimately, G1's chemical properties are found to resemble other M31 GCs, though it also shares some similarities with extragalactic nuclear star clusters.
\end{abstract}

Key words: galaxies: individual(M31) — galaxies: abundances — galaxies: star clusters: individual(G1) — globular clusters: general — galaxies: evolution

\section{INTRODUCTION}

Historically, globular clusters (GCs) were once regarded as simple stellar populations, i.e., dark-matter-free, chemically-homogeneous spheroids that were fundamentally distinct from the more chemically complex galaxies. It is now widely accepted, however, that GCs are not simple: all GCs in the Milky Way and its satellites show some evidence for star-to-star chemical inhomogeneity,

*Based on observations obtained with the Hobby-Eberly Telescope, which is a joint project of the University of Texas at Austin, the Pennsylvania State University, Ludwig-MaximiliansUniversität München, and Georg-August-Universität Göttingen. †E-mail: sakaricm@sfsu.edu from variations in carbon and nitrogen (seen in all massive GCs, including the intermediate-age LMC GCs; e.g., Hollvhead et al. 2017, 2019), to variations in sodium and oxygen (seen in all classical, old GCs; e.g., Carretta et al. 2009), to variations in heavier elements in a handful of GCs, including neutron-capture elements (Sneden et al. 1997; Roederer 2011) and even iron. Iron is one of the more puzzling elements that can vary within GCs, as iron spreads are generally interpreted as a signature of multiple bursts of star formation. However, some of the most massive GCs exhibit clear spreads in $[\mathrm{Fe} / \mathrm{H}]$, including the most massive Milky Way (MW) GC, $\omega$ Cen, which shows at least four discrete populations with an iron spread of $\sim 2$ dex (Freeman \& Rogers 1975; Johnson \& Pilachowski 2010; Villanova et al. 2014). There are at least five more 
of these "iron-complex" GCs in the MW with clear, significant iron spreads (Carretta et al. 2010; Marino et al. 2011a, 2015; Yong et al. 2014; Johnson et al. 2017). These iron-complex GCs also host $\mathrm{Na} / \mathrm{O}$ variations, just like the classical GCs; it is uncertain how these iron-complex GCs fit in with the general GC population.

A potential explanation for the iron-complex GCs is that they were once the nuclear star clusters (NSCs) of dwarf galaxies which have since been accreted into the MW. In the NSC framework, the iron spreads are then caused by ongoing in situ star formation, mergers of multiple classical GCs, or some mixture of the two scenarios (see the review by Neumaver et al. 2020). There is some observational evidence to suggest that the iron-complex GCs did originate in dwarf galaxies. One of the iron-complex GCs, M54 (Carretta et al. 2010), lies very close to the expected core of the Sagittarius dwarf spheroidal, a galaxy which is actively being accreted into the MW halo (Ibata et al. 1995). Johnson et al. (2017) also found several stars in M19 with low $[\alpha / \mathrm{Fe}]$, a common signature of metal-rich dwarf galaxy stars (e.g., Tolstoy et al. 2009). Most of the iron-complex GCs in the MW, however, are dominated by metal-poor, $\alpha$-enhanced stars that follow the MW's standard chemical evolution track (e.g., Marino et al. 2015; Johnson et al. 2017), making it difficult to identify accreted GCs through chemical abundance analyses. Other groups have identified tidal streams or field star populations around the iron-complex GCs (e.g., Ibata et al. 2019), and several iron-complex GCs have been linked to streams and dwarf galaxies, including Gaia-Enceladus (Helmi et al. 2018; Massari et al. 2019). Unraveling the mystery of the iron-complex GCs requires identifying more clusters and studying their detailed chemical abundances and kinematics.

Massive GCs are not unique to the MW; other galaxies have GCs that are even more massive than $\omega$ Cen , including M31. One such cluster is G1 (also known as Mayall II; Mavall \& Eggen 1953). G1's total mass has been estimated to be $\sim 7-17 \times 10^{6} \mathrm{M}_{\odot}$, making it at least twice as massive as $\omega$ Cen (Meylan et al. 2001). Photometric evidence suggests that G1 is also an iron-complex GC: the width of its red giant branch (RGB) in an Hubble Space Telescope (HST) CMD indicates an $[\mathrm{Fe} / \mathrm{H}]$ dispersion of $0.1-0.5 \mathrm{dex}$ (Meylan et al. 2001; Nardiello et al. 2019) 11 G1 has also gained some notoriety for being the potential host of an intermediate-mass black hole (Gebhardt et al. 2002, 2005), though Miller-Jones et al. 2012 argue that the X-ray and radio data are more indicative of a low-mass X-ray binary. Baumgardt et al. (2003) argue that G1's dynamics match models for a GC-GC merger, which would be consistent with an origin as a NSC.

G1 also has indications that it may have originated in a dwarf galaxy. It seems to be a relatively metal-rich GC, at $[\mathrm{Fe} / \mathrm{H}] \sim-0.7$ to -1 (Rich et al. 1996; Mevlan et al. 2001; Perina et al. 2012) despite its location in the outer halo $\left(R_{\text {proj }}=34.7 \mathrm{kpc}\right.$ from the centre of M31; Mackey et al.
2019). Simulations by Bekki \& Chiba (2004) confirm that G1 could indeed have been created by stripping a nucleated dwarf of its outer envelope of stars. However, tidal debris has not been found around G1 (Reitzel et al. 2004). Mackev et al. (2010) also noted that G1 appears to lie within a group of GCs on the western side of M31, known as "Association 2," but Velianoski et al. (2014) found that G1's radial velocity did not match the other GCs. G1's presence in the outer halo is therefore somewhat of a mystery; there are strong indications that it may have been a NSC, yet there is no sign of the rest of the galaxy. There are also other similarly massive clusters in M31, though none are obviously in the outer halo (but see Perina et al. 2012). Detailed chemical abundances of a wide variety of elements from stars in this moderately metal-poor cluster can shed light on its possible status as a GC and as a former dwarf galaxy NSC. However, G1's distance renders its brightest stars too faint for high resolution, high $\mathrm{S} / \mathrm{N}$ spectroscopy, since the brightest stars have $V \sim 22 \mathrm{mag}$.

Fortunately, G1 can be studied through high resolution integrated light (IL) spectroscopy, where a single spectrum is obtained from an entire stellar population. The capabilities and limitations of high-resolution IL spectroscopy have been thoroughly laid out by McWilliam \& Bernstein (2008), Colucci et al. (2009, 2011, 2012, 2014), and Sakari et al. (2013, 2014, 2015, 2016). Briefly, high resolution IL spectroscopy can produce flux-weighted average abundances of many elements, including $\mathrm{Fe}, \mathrm{Mg}$, $\mathrm{Ca}$, Ba, and Eu. Tests with Milky Way GCs have demonstrated that IL abundances match the values from individual stars: when the elements do not vary within a cluster, these integrated abundances represent the primordial values; otherwise, the integrated abundances fall within the observed ranges (e.g., McWilliam \& Bernstein 2008; Sakari et al. 2013; Colucci et al. 2017). Though G1's putative Fe spread complicates these analyses, the uncertainties can be robustly quantified, and the possibilities for identifying an Fe spread can be explored - this will be the subject of a forthcoming paper.

This paper therefore presents the first detailed chemical abundances in the massive M31 GC, G1. A medium resolution calcium II triplet (CaT) spectrum is also presented as an independent verification of the average cluster metallicity. Section 2 presents the observations and data reduction, while Section 3 discusses the identification of an appropriate isochrone to model the underlying stellar population and the resulting chemical abundances. G1's status as a GC, as a potential NSC, and as a member of M31's outer halo are then discussed in Section 4 Given the likelihood of intra-cluster abundance spreads within G1, the systematic offsets resulting from undetected abundance spreads are quantified in a forthcoming paper (Sakari et al., in prep.).
1 Mevlan et al. (2001) estimated $\Delta[\mathrm{Fe} / \mathrm{H}]=0.4-0.5 \mathrm{dex}$, but Nardiello et al. (2019) have subsequently revised this estimate down to $\Delta[\mathrm{Fe} / \mathrm{H}] \sim 0.15$ dex. 
Table 1. Information about G1.

\begin{tabular}{|c|c|c|}
\hline$\overline{\text { Parameter }}$ & Value & Note/Reference \\
\hline$\overline{\mathrm{RA}(\mathrm{J} 2000)}$ & $00: 32: 46.536$ & 1 \\
\hline Dec $(\mathrm{J} 2000)$ & $+39: 34: 40.67$ & 1 \\
\hline$V_{\text {tot }}$ & 13.81 & 2 \\
\hline Observation Dates & $\begin{array}{l}2007 \text { Aug } 8, \\
2008 \text { Oct } 29\end{array}$ & HET Spectrum \\
\hline Total exposure time & $5400 \mathrm{~s}$ & \\
\hline $\mathrm{S} / \mathrm{N}(5000 \AA)^{a}$ & 230 & \\
\hline $\mathrm{S} / \mathrm{N}(6500 \AA)^{a}$ & 320 & \\
\hline$v_{\text {helio }}\left(\mathrm{km} \mathrm{s}^{-1}\right)$ & -349.7 & \\
\hline$\sigma_{V}\left(\mathrm{~km} \mathrm{~s}^{-1}\right)$ & $23.9 \pm 2.0$ & \\
\hline Observation Dates & 2014 Oct 5 & APO Spectrum \\
\hline Exposure time & $900 \mathrm{~s}$ & \\
\hline $\mathrm{S} / \mathrm{N}(8600 \AA)^{a}$ & 216 & \\
\hline$v_{\text {helio }}\left(\mathrm{km} \mathrm{s}^{-1}\right)$ & -335.3 & \\
\hline Literature $v_{\text {helio }}\left(\mathrm{km} \mathrm{s}^{-1}\right)$ & $-335 \pm 5$ & 3 \\
\hline Literature $\sigma_{V}\left(\mathrm{~km} \mathrm{~s}^{-1}\right)$ & $21.4 \pm 1.3$ & Uncorrected $^{b} ; 4$ \\
\hline & $24.5 \pm 1.5$ & Corrected $^{b} ; 4$ \\
\hline \multicolumn{3}{|l|}{ References: } \\
\hline \multicolumn{3}{|c|}{$\begin{array}{l}\text { 1: SIMBAD; } 2 \text { Galleti et al. (2009) } ; 3 \text { : Velianoski et al. (2014) } \\
\text { 4: Cohen (2006) } \\
a \text { S/N ratios are per resolution element. } \\
b \text { Cohen (2006) applied a correction to the velocity dispersion } \\
\text { that accounted for the aperture size. }\end{array}$} \\
\hline
\end{tabular}

\section{OBSERVATIONS AND DATA REDUCTION}

\subsection{High Resolution Spectrum}

The G1 data were obtained at McDonald Observatory in Fort Davis, TX using the High Resolution Spectrograph (HRS; Tull 1998) on the Hobby-Eberly Telescope (HET; Ramsev et al. 1998; Shetrone et al. 2007). Observations were carried out in 2007 and 2008 (see Table 1). A spectral resolution of $R=15,000$ was chosen, since G1's large velocity dispersion renders a higher resolution unnecessary. The $600 \mathrm{gr} / \mathrm{mm}$ cross disperser was used at a central wavelength of $5822 \AA$; as a result the wavelength coverage is $\sim 4800-5790 \AA$ and $\sim 5830-6820 \AA$. Simultaneous sky spectra were obtained with adjacent sky fibres located $10^{\prime \prime}$ from the cluster centre. G1's half-light radius $\left(r_{h}=1.73^{\prime \prime}\right.$; Ma et al. 2007) is less than the size of the HRS fibre $\left(3^{\prime \prime}\right)$.

However, note that the sky fibres are contaminated by star light, at least one of them by a bright, foreground star. For that reason, sky spectra were not subtracted from the target spectrum. Since G1 is bright, individual exposures were short (15 minutes), the spectra do not extend far into the blue, and the remaining sky fiber had minimal flux, the sky continuum will not have a significant effect on the final IL spectrum or the subsequent analysis.

The data reduction was performed in the Image Reduction and Analysis Facility program (IRAF) 2 Standard data reduction procedures for echelle spectra were adopted;

2 IRAF is distributed by the National Optical Astronomy Observatory, which is operated by the Association of Universities for Research in Astronomy, Inc., under cooperative agreement with the National Science Foundation. since the cluster is so bright there is no need for variance weighting to remove cosmic rays, unlike in previous IL analyses (Sakari et al. 2013). The individual exposures were shifted to the rest frame through cross-correlations with the high resolution, high S/N Arcturus spectrum from Hinkle et al. (2003) 3 The final, heliocentric radial velocity is shown in Table 1 After the spectra were shifted to the rest frame, individual exposures were combined with average sigma-clipping routines. The cluster velocity dispersion (also given in Table 1) was determined through a cross-correlation with Arcturus, using a calibrated relationship between the full-width at half maximum and the velocity dispersion (Alpaslan 2009; Sakari et al. 2013). No correction was made to account for the aperture size (see Cohen 2006).

The continuum was normalized carefully, since the moderate spectral resolution and large velocity dispersion can lead to line blanketing in regions with strong absorption. The blaze function of the orders was removed with loworder polynomial fits and the individual orders were then combined. Because all lines are fit with spectrum syntheses, continuum problems are not likely to be a significant problem in smaller $10 \AA$ regions.

Figure 1 shows the HRS IL spectrum of G1 compared to the IL spectrum of 47 Tuc, where the high-resolution 47 Tuc spectrum from McWilliam \& Bernstein (2008) has been broadened to match the velocity dispersion of G1. The two spectra are generally quite similar, although there are a few regions where they differ, either due to continuum issues or differing line strengths. A more detailed comparison will be discussed further below.

\subsection{CaT Spectrum}

A CaT spectrum of G1 was obtained in 2014 with the Dual Imaging Spectrograph (DIS) on the Astrophysical Research Consortium $3.5 \mathrm{~m}$ telescope at Apache Point Observatory in New Mexico. The observational program for these IL CaT measurements is described in Sakari \& Wallerstein (2016). The spectral range from $\sim 8000-9100 \AA$ was covered by the red camera. The R1200 grating and $1.5^{\prime \prime}$ slit give a spectral resolution of $R \sim 4000$ in the CaT region, which is sufficient to detect the strong CaT lines. The length of the slit $\left(6^{\prime}\right)$ fully covered G1 past its tidal radius (22" ; Ma et al. 2007). An exposure time of 15 min yielded a $\mathrm{S} / \mathrm{N} \sim 216$ per resolution element at $8600 \AA$.

The CaT data were reduced in IRAF, as described in Sakari \& Wallerstein (2016), utilizing variance weighting, sky subtraction with aligned sky lines, and careful continuum normalization with a low-order polynomical. As with the high resolution spectrum, the heliocentric radial velocity was determined from a cross-correlation with the Arcturus spectrum, though in this case the Arcturus spectrum was degraded to the resolution of DIS. The CaT heliocentric radial velocity agrees well with the high resolution

3 ftp://ftp.noao.edu/catalogs/arcturusatlas/ 


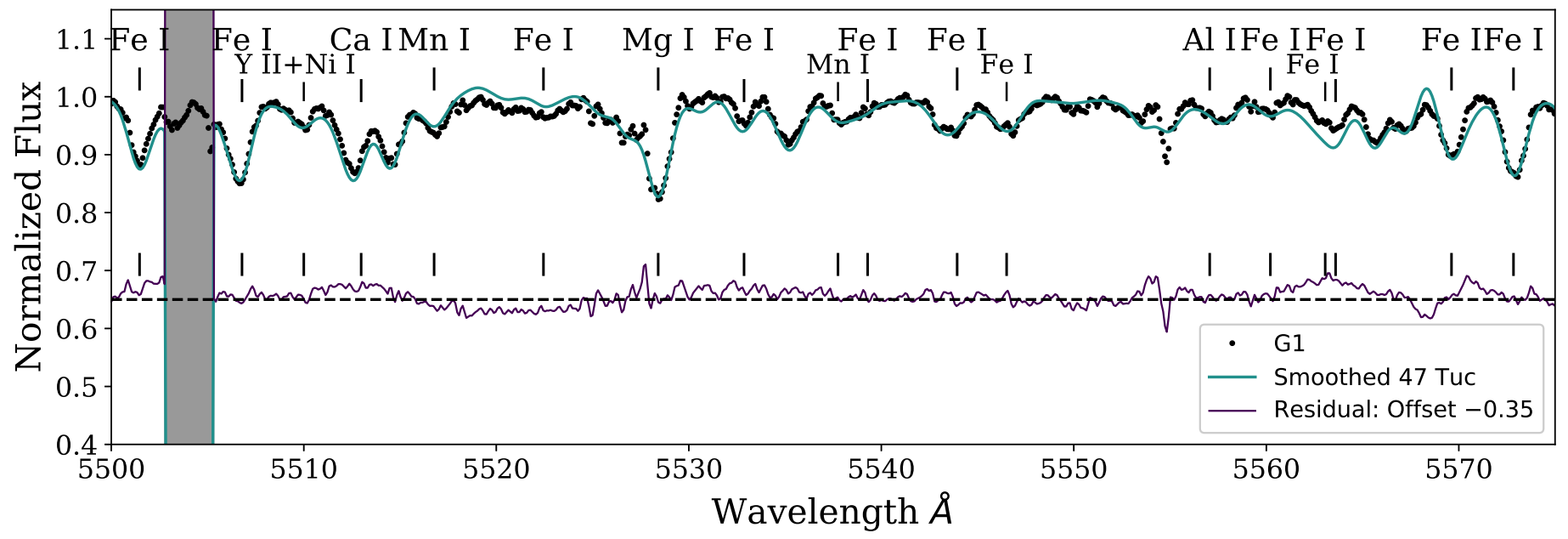

Figure 1. A comparison between the optical, high-resolution IL spectra of G1 (points) and 47 Tuc (the thick green line; from McWilliam \& Bernstein 2008), where the 47 Tuc spectrum has been smoothed to the velocity dispersion of G1. The thin purple line (arbitrarily offset below the two spectra) shows the residuals. The grey region shows a bad region of the 47 Tuc spectrum.

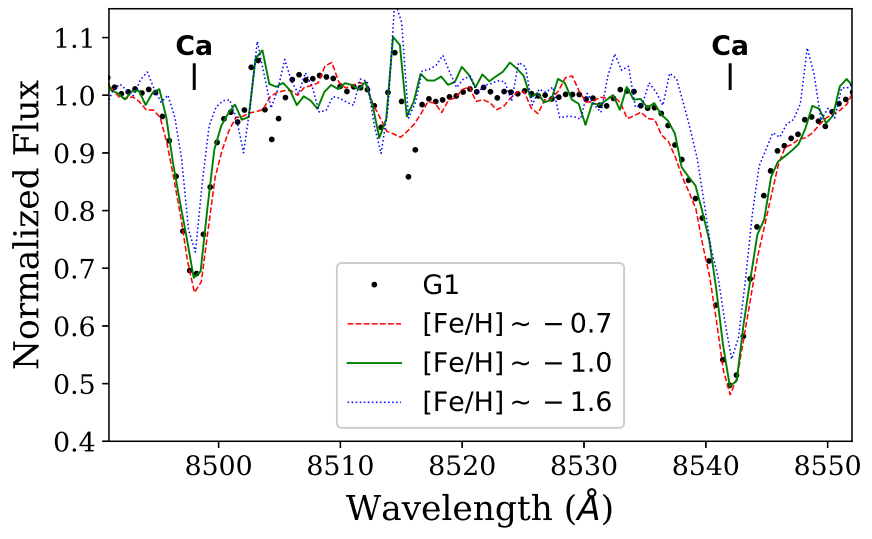

Figure 2. The first two CaT lines in the G1 spectrum (black dots), compared to three spectra of M31 GCs from Sakari \& Wallerstein (2016): B225 (with a CaT $[\mathrm{Fe} / \mathrm{H}]=-0.7), \mathrm{B} 182([\mathrm{Fe} / \mathrm{H}]=-1.0)$, and B012 $([\mathrm{Fe} / \mathrm{H}]=-1.6) . \mathrm{G} 1$ 's CaT lines are most similar to the B182 spectrum.

value and the literature value from Cohen (2006). A portion of the CaT spectrum is shown in Figure 2 along with three other M31 GCs (from Sakari \& Wallerstein 2016).

\section{A TRADITIONAL INTEGRATED LIGHT ANALYSIS: ONE AGE, ONE [FE/H]}

The first step of a traditional high-resolution IL spectral analysis, according to the methods of McWilliam \& Bernstein (2008), is to create a HertzsprungRussell Diagram (HRD) for the cluster's underlying stellar population. Without high quality, resolved photometry that covers the full cluster past the main sequence turnoff, the population must be modeled with an isochrone, for which the age and metallicity are the primary parameters. A complex cluster like G1 could have stars with a range of ages and metallicities; however, the simplest initial step is to identify a single age and metallicity to represent the entire G1 population. The resulting effects of abundance spreads, including the effects on the adopted models, will be investigated in a subsequent paper. An initial estimate of the metallicity is first obtained from the medium-resolution CaT spectra (Section 3.1.1); the final parameters are then determined from a detailed inspection of the Fe I lines (Section 3.1.2). Abundances and systematic errors are then presented in Sections 3.2 and 3.3 .

\subsection{Age and Metallicity}

\subsection{1 $[\mathrm{Fe} / \mathrm{H}]$ from the calcium-II triplet}

IL CaT features have been used for decades to infer GC metallicities (e.g., Armandroff \& Zinn 1988; Foster et al. 2010, 2011; Usher et al. 2012). Sakari \& Wallerstein (2016) present a comparison of CaT strengths with highresolution IL $[\mathrm{Fe} / \mathrm{H}]$ in $\mathrm{M} 31 \mathrm{GCs}$, verifying that the IL $\mathrm{CaT}$ is a tracer of cluster metallicity, at least for GCs older than $\sim 3$ Gyr and with $[\mathrm{Fe} / \mathrm{H}] \lesssim-0.24$ The precise relationship between $\mathrm{CaT}$ strength and $[\mathrm{Fe} / \mathrm{H}]$ depends strongly on how the lines are measured and how the continuum is treated. In this paper, G1's CaT spectrum is analyzed in the same way as the other M31 GCs in Sakari \& Wallerstein (2016): its observed spectrum is

\footnotetext{
4 Sakari \& Wallerstein (2016) focused on trends with [Fe/H], noting that a cluster's $[\mathrm{Ca} / \mathrm{Fe}]$ can also affect the strength of the CaT lines. Usher et al. (2019) find that the CaT lines may track $[\mathrm{Ca} / \mathrm{H}]$ better than $[\mathrm{Fe} / \mathrm{H}]$.
} 
fit with a linear combination of template spectra (from stars that were observed with the same instrumental setup) utilizing the penalized pixel-fitting code pPXF (Cappellari \& Emsellem 2004). Voigt profiles were then fit to each of the three lines to determine EWs, using the pymodelfit program 5 The relationships between CaT EW and $[\mathrm{Fe} / \mathrm{H}]$ from Table 2 in Sakari \& Wallerstein (2016) were then utilized to determine G1's CaT metallicity, using all three CaT lines. The CaT-based metallicity is found to be $[\mathrm{Fe} / \mathrm{H}]=-0.85 \pm 0.10$. Figure 2 compares G1's CaT spectrum with three other M31 GCs (from Sakari \& Wallerstein 2016), showing that G1 does indeed appear to be moderately metal-poor.

\subsubsection{Age and Metallicity from Fe I Lines}

For the high-resolution analysis, the Bag of Stellar Tracks and Isochrones (BaSTI) models from the Teramo group (Pietrinferni et al. 2004, 2006) were used to synthesize G1's underlying population, assuming an extended asymptotic giant branch (AGB) with a mass loss parameter of $\eta=0.2$ (see Sakari et al. 2014 for discussions of the effects of AGB morphology on IL analyses). The isochrones were populated using a Kroupa (2002) initial mass function (IMF) and the resulting HRDs were binned so that each box contains $3 \%$ of the total flux. Alpha-enhanced (AODFNEW) Kurucz model atmospheres (Castelli \& Kurucz 2004) were assigned to each box, using the $T_{\text {eff }}$ and $\log g$ interpolation scheme from McWilliam \& Bernstein (2008). Following the procedure from McWilliam \& Bernstein (2008), Colucci et al. (2009, 2011, 2014, 2017), and Sakari et al. (2013, 2015, 2016), an appropriate isochrone age and metallicity were selected based on the abundances from the Fe I lines. As explained in McWilliam \& Bernstein (2008), this technique is based on the method used to derive atmospheric parameters for individual stars, and requires a sample of Fe I lines that span a range of wavelengths, reduced equivalent widths (REW) 7 and excitation potentials (EPs). Since nearly every line is a blend in G1's spectrum, equivalent widths cannot be measured for the Fe I lines; instead, each Fe I line was synthesized with 20 different isochrones spanning ages of $6,8,10,12$, and 14 Gyr and metallicities of $[\mathrm{Fe} / \mathrm{H}]=-0.6,-0.7,-1.01$, and -1.31 dex. The synpop routine in the 2015 version of the Local Thermodynamic Equilibrium (LTE) line analysis code MOOG (Sneden 1973) was used to synthesize spectral lines. The linelists were generated with the linemake code 8 including hyperfine structure (HFS) and isotopic splitting as well as molecular lines from $\mathrm{CH}, \mathrm{C}_{2}$, and $\mathrm{CN}$.

Many of the spectral lines that can be measured in the

\footnotetext{
5 https://pythonhosted.org/PyModelFit/

6 http://kurucz.harvard.edu/grids.html

$7 \mathrm{REW}=\log (\mathrm{EW} / \lambda)$.

8 https://github.com/vmplacco/linemake
}

Table 2. Parameters of the adopted single-population isochrones, as determined from the IL spectra.

\begin{tabular}{lccc}
\hline & \multicolumn{3}{c}{ Isochrone Parameters } \\
Cluster & {$[\mathrm{Fe} / \mathrm{H}]$} & Age $(\mathrm{Gyr})$ & {$[\alpha / \mathrm{Fe}]$} \\
\hline G1 & -1.01 & 10 & +0.4 \\
47 Tuc & -0.70 & 10 & +0.4 \\
\hline
\end{tabular}

G1 spectrum are necessarily fairly strong, due to the spectral resolution. In general, strong lines are undesirable for model atmospheres analysis because of difficulties modeling the outer layers of the model atmospheres (McWilliam et al. 1995). Unfortunately, the desirable weak lines are not easily detectable in the broadened G1 spectrum. Line strengths were therefore restricted to $\mathrm{REW}<-4.6$. This limit is higher than the $\mathrm{REW}=-4.7$ limit recommended by McWilliam et al. (1995); however, comparisons with the same lines in 47 Tuc (Section 3.2.7) demonstrate that selecting these higher REW lines does not lead to significant systematic effects in the abundances. It is also worth remembering that although a REW limit is placed on the IL spectral lines, the individual HRD bins could have lines that are stronger than this REW limit; the usage of a REW limit is therefore not straight-forward in IL analyses.

Given G1's similarity to 47 Tuc, the broadened 47 Tuc spectrum was re-analyzed, using the isochrone parameters identified by Sakari (2014, see Table 2). The only lines that were utilized for this analysis are lines that are also measured in the G1 spectrum. The G1 Fe abundances were then considered differentially with respect to the 47 Tuc lines. Given that strong lines are used both for 47 Tuc and G1, this differential analysis should reduce the effects from uncertain atomic data, damping constants, line blends, etc. The final trends in $[\mathrm{Fe} \mathrm{I/H}]$, relative to 47 Tuc, with respect to wavelength, REW, and EP were then calculated for the twenty isochrones, over a range of ages and metallicities. The resulting slopes are shown in Figure 3. The offset in $[\mathrm{Fe} / \mathrm{H}]$ between the input isochrone and the average of the MOOG output for the synthesized lines is shown in Figure 4 Although a number of isochrones produce reasonably flat trends in $[\mathrm{Fe} / \mathrm{H}]$ with wavelength, REW, and EP, the best match is for an isochrone with $[\mathrm{Fe} / \mathrm{H}]=-1.01$ dex and an age of $10 \mathrm{Gyr}$ (see Figure 5). Figures 3 and 4 also show that the cluster age is poorly constrained, which is consistent with previous results from Colucci et al. (2009, 2014), Sakari et al. (2015), and other papers, who found that the optical Fe I lines were not very sensitive to the cluster age.

The average metallicity for G1 is slightly more metal-poor than 47 Tuc $([\mathrm{Fe} \mathrm{I} / \mathrm{H}]=-0.98 \pm 0.05$ dex compared to $[\mathrm{Fe} \mathrm{I} / \mathrm{H}]=-0.76 \pm 0.03 \mathrm{dex})$. Figure 6 shows that several of the lines in G1 are indeed weaker than the lines in 47 Tuc, supporting a lower metallicity. The high-resolution spectroscopic metallicity is also lower than the IL CaTbased $[\mathrm{Fe} / \mathrm{H}]$ (Section 3.1.1). This discrepancy may reflect $\alpha$-enhancement in G1. With a large sample of GCs in the Milky Way and nearby dwarf galaxies, Usher et al. (2019) find that the CaT strength is a better tracer of $[\mathrm{Ca} / \mathrm{H}]$ than $[\mathrm{Fe} / \mathrm{H}]$. For a cluster with $[\mathrm{Ca} / \mathrm{Fe}]=+0.4$, they find 

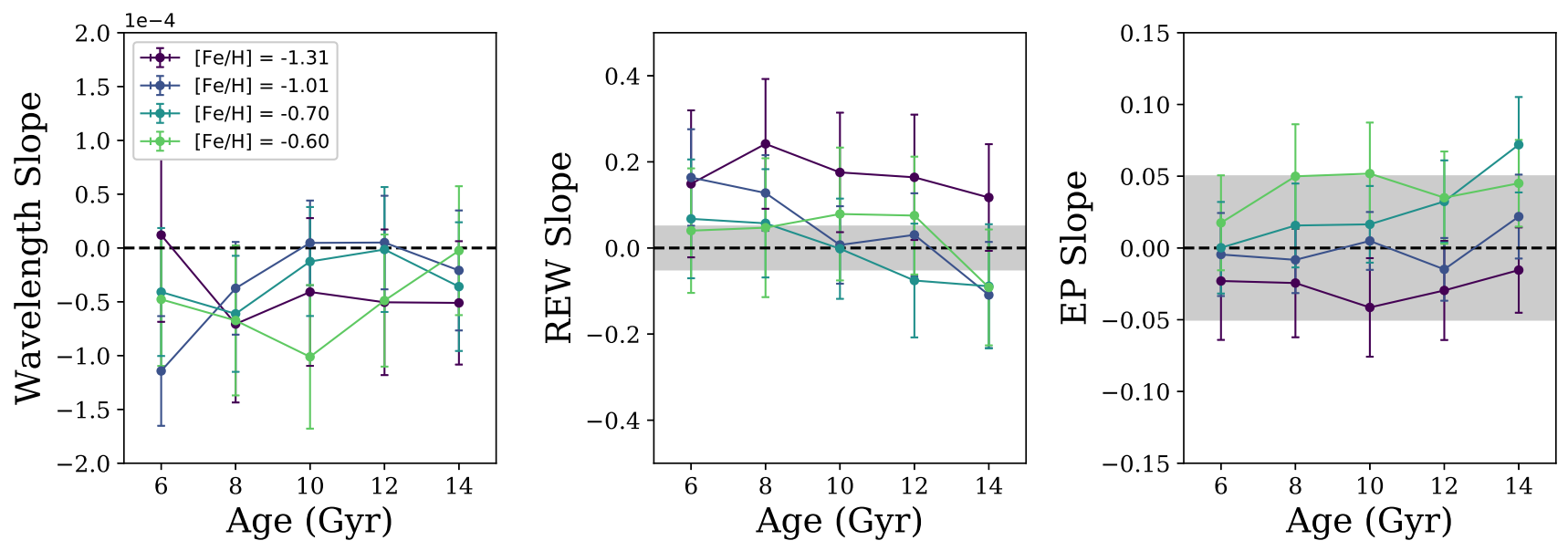

Figure 3. The overall slopes of the distributions in wavelength, REW, and EP versus Fe I abundance as a function of isochrone age (in Gyr). Four different isochrone metallicities are shown: $[\mathrm{Fe} / \mathrm{H}]=-1.31$ (purple), $[\mathrm{Fe} / \mathrm{H}]=-1.01$ (dark blue), $[\mathrm{Fe} / \mathrm{H}]=-0.70($ light blue), and $[\mathrm{Fe} / \mathrm{H}]=-0.60$ (green). The grey bands show slopes of \pm 0.05 in REW and EP, which are considered to be acceptably flat slopes. Some of the variations with age amongst isochrones of the same metallicity occur as lines move in or out of the acceptable line strength limit of REW $<-4.6$.

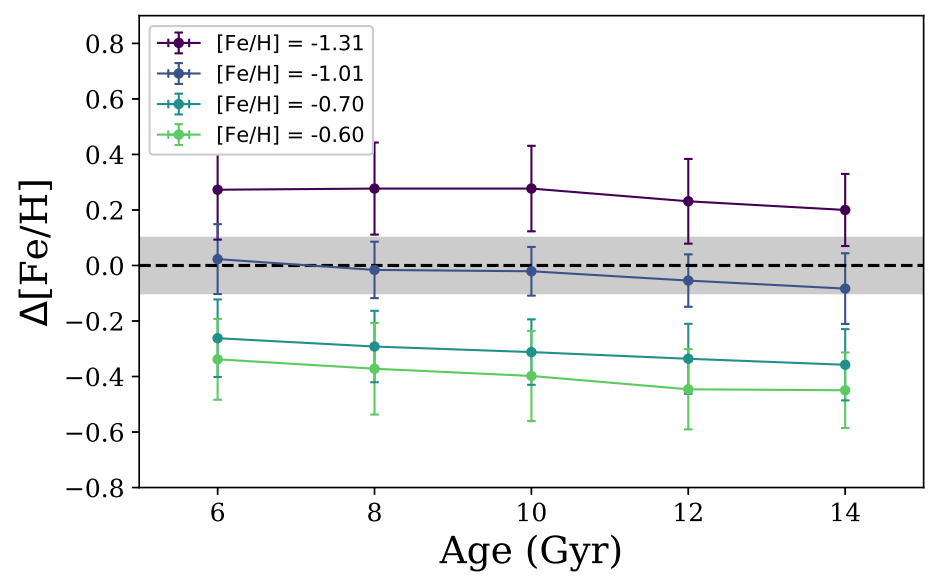

Figure 4. Offsets between the average $[\mathrm{Fe} \mathrm{I} / \mathrm{H}]$ and the input isochrone $[\mathrm{Fe} / \mathrm{H}]$, as a function of isochrone age (in Gyr). Lines are as in Figure 3 The grey bar shows a difference of \pm 0.1 dex.

that an $[\mathrm{Fe} / \mathrm{H}]$ derived from the CaT could be about 0.1 dex higher than the value from a high-resolution analysis. If G1 is similarly $\alpha$-enhanced (see Section 3.2.4), then we might expect that its $\mathrm{CaT}[\mathrm{Fe} / \mathrm{H}]$ has been over-estimated by 0.1 dex.

\subsubsection{G1's Metallicity: Comparisons with Previous Results}

The spectroscopic metallicity of $[\mathrm{Fe} / \mathrm{H}]=-0.98 \pm 0.05$ is consistent with previous photometric $[\mathrm{Fe} / \mathrm{H}]$ estimates for G1. Based on measurements of the RGB slope in the HST photometry and comparisons with 47 Tuc fiducials, Rich et al. (1996) and Meylan et al. (2001) find that G1 should have an average $[\mathrm{Fe} / \mathrm{H}]$ around -0.95 . Based on a similar analysis, Federici et al. (2012) find $[\mathrm{Fe} / \mathrm{H}]=-$ 0.90. Meylan et al. (2001) further concluded that there could be a wide Fe spread of $0.4-0.5$ dex based on the width of G1's RGB. Nardiello et al. (2019) conducted a subsequent re-analysis of the HST photometry, focusing on signs of abundance spreads within G1. Based on comparisons with the BaSTI isochrones they adopted a standard red $\mathrm{RGB}$ sequence with $[\mathrm{Fe} / \mathrm{H}]=-0.70$ and found that a much smaller spread (down to $[\mathrm{Fe} / \mathrm{H}]=-0.85$ ) was consistent with the observed width of the RGB - they argue that the $\Delta[\mathrm{Fe} / \mathrm{H}]$ could be slightly smaller if there are additional helium or CNO variations within the cluster. G1's primarily red horizontal branch also indicates that the dominant population is fairly metal-rich-however, many authors (Rich et al. 1996; Mevlan et al. 2001; Perina et al. 2012; Nardiello et al. 2019) have noted the presence of bluer horizontal branch stars, which further indicates that there may be a helium spread within G1.

The spectroscopic $[\mathrm{Fe} / \mathrm{H}]$ derived here also agrees with previous spectroscopic analyses. Reitzel et al. (2004) obtained CaT spectra of individual M31 field stars, including at least one star that is a likely member of G1 based on its radial velocity; this likely member has a CaT-based metallicity of $[\mathrm{Fe} / \mathrm{H}]=-0.74$. Other IL spectroscopic analyses at lower-resolution also find similar metallicities. The earliest abundance analysis by van den Bergh (1969) found $[\mathrm{Fe} / \mathrm{H}]=-0.8$, while subsequent analyses found similar results (e.g., Huchra et al. 1991 found $[\mathrm{Fe} / \mathrm{H}]=-1.01)$. The Revised Bologna Catalog $(\mathrm{RBC}) 9$ reports $[\mathrm{Fe} / \mathrm{H}]=-0.73 \pm 0.15$, based on a Lick index analysis (Galleti et al. 2009)-however, Colucci et al. (2014) note that the Lick index $[\mathrm{Fe} / \mathrm{H}]$ ratios of their sample of M31 GCs are slightly higher than the highresolution values for clusters at $[\mathrm{Fe} / \mathrm{H}]=-1$. The

\footnotetext{
9 http://www.bo.astro.it/M31/
} 

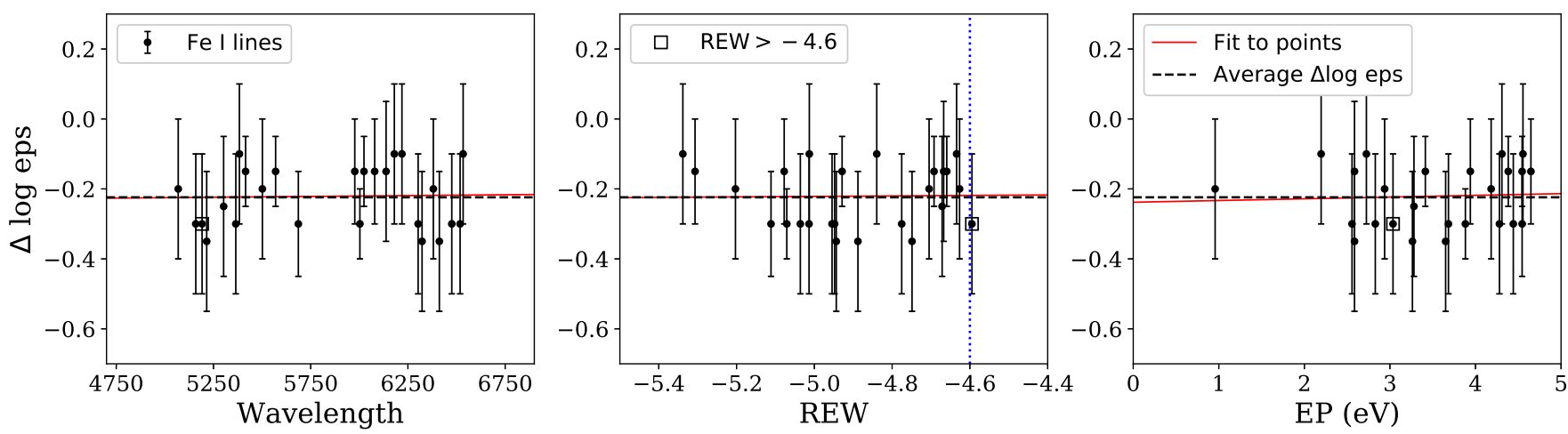

Figure 5. The $\Delta[\mathrm{Fe} / \mathrm{H}]$ offsets as a function of wavelength, REW, and EP between $\mathrm{G} 1$ and 47 Tuc for individual Fe I lines (circles) when the $[\mathrm{Fe} / \mathrm{H}]=-1.01,10 \mathrm{Gyr}$ isochrone is adopted for $\mathrm{G} 1$. The vertical dotted blue line shows $\mathrm{REW}=-4.6$ limit; the square shows one spectral line that falls above this limit and is therefore not included in the fits. The dashed black lines show the average $\Delta[\mathrm{Fe} / \mathrm{H}]$, while the solid red lines show the fits for each panel.
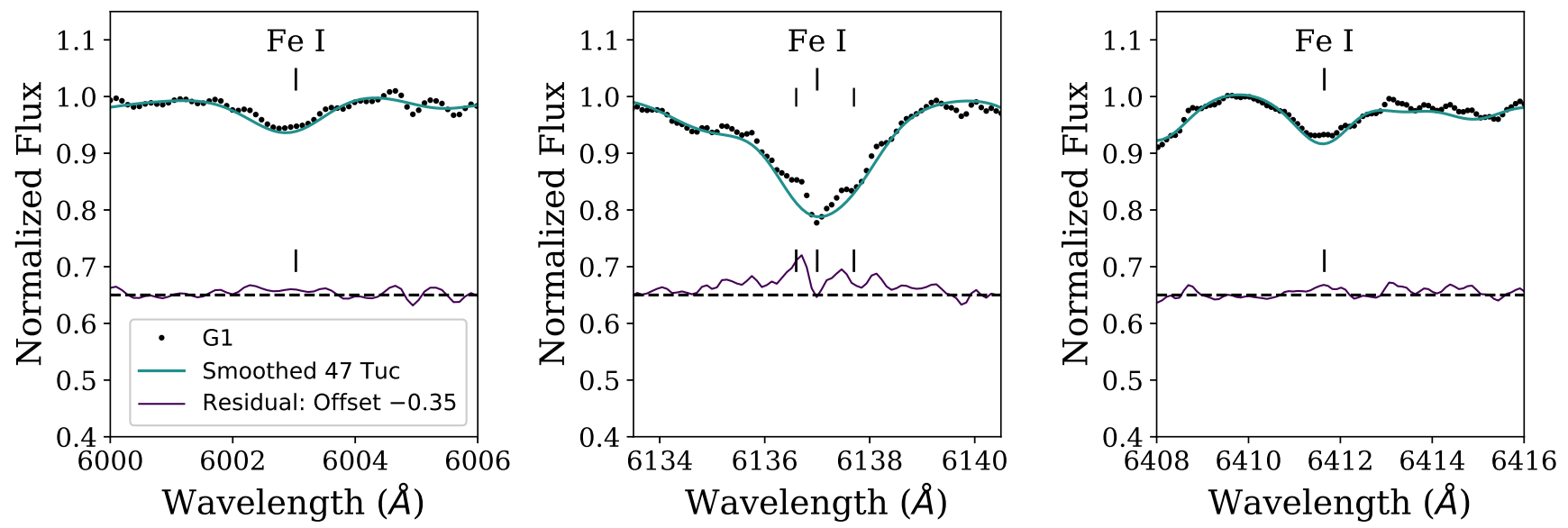

Figure 6. A comparison between three Fe I features in the optical, high-resolution IL spectra of G1 (points) and 47 Tuc (bold green line; from McWilliam \& Bernstein 2008), where the 47 Tuc spectrum has been smoothed to the velocity dispersion of G1. The small vertical lines show the locations of individual spectral lines. The thin purple line (arbitrarily offset below the two spectra) shows the residuals. These small offsets indicate that G1 is slightly more metal-poor than 47 Tuc.

spectroscopic value in this paper is therefore generally consistent with other values from the literature.

Of course, the possibility of an iron spread in G1 makes it difficult to interpret a single IL value. The metallicity of G1 will be discussed more in Section 3.2 .1 and in a forthcoming paper.

\subsection{Detailed Abundances of G1}

The abundances of other elements were determined via spectrum syntheses in MOOG, using the single stellar population isochrone parameters in Table 2 Note that for IL analyses Sakari et al. (2013) found that it was better to use line-to-line differential abundances with respect to solar abundances derived with the same techniques, atomic data, etc. That technique has not been used here, however, since many of the lines that are detectable in the G1 spec- trum are too strong in the solar spectrum. Instead, 47 Tuc is used as a reference. Comparisons with literature values for 47 Tuc are given in Section 3.2.7.

The single population $[\mathrm{Fe} / \mathrm{H}]$ and $[\mathrm{X} / \mathrm{Fe}]$ abundance ratios are shown in Table 3, individual abundances per spectral line are given in Appendix A Abundances for 47 Tuc are also shown in Table 3 , along with the abundance ratio differences between G1 and 47 Tuc.

\subsubsection{Iron}

The $25 \mathrm{Fe}$ I lines used for this analysis span a range of wavelengths, EPs, and line strengths, as discussed in Section 3.1.2 Two Fe II lines, at 5534 and $6456 \AA$, are also detectable in G1's spectrum. The resulting [Fe II/H] is 0.15 dex higher than the $[\mathrm{Fe} \mathrm{I} / \mathrm{H}]$ ratio. $\mathrm{A}+0.06 \mathrm{dex}$ offset between Fe II and Fe I is also found in 47 Tuc. 
Table 3. Isochrone-based IL Abundances for G1 and 47 Tuc.

\begin{tabular}{|c|c|c|c|c|c|}
\hline \multirow{2}{*}{ Element } & \multicolumn{2}{|l|}{ G1 } & \multicolumn{2}{|l|}{47 Tuc } & \multirow{2}{*}{$\begin{array}{l}\text { Abundance } \\
\text { (G1-47 Tuc) }\end{array}$} \\
\hline & Abundance & $N$ & Abundance & $N$ & \\
\hline$\overline{[\mathrm{Fe} \mathrm{I} / \mathrm{H}]}$ & $-0.98 \pm 0.05$ & 25 & $-0.76 \pm 0.03$ & 25 & -0.22 \\
\hline$[\mathrm{Fe} \mathrm{II} / \mathrm{H}]$ & $-0.83 \pm 0.10$ & 2 & $-0.70 \pm 0.10$ & 2 & -0.13 \\
\hline$[\mathrm{C} \mathrm{I} / \mathrm{Fe}]$ & $<0.17$ & 4 & $<-0.44$ & 4 & - \\
\hline$[\mathrm{Na} \mathrm{I} / \mathrm{Fe} \mathrm{I}]$ & $0.60 \pm 0.13$ & 4 & $0.38 \pm 0.05$ & 4 & +0.22 \\
\hline$[\mathrm{Mg} \mathrm{I} / \mathrm{Fe} \mathrm{I}]$ & $0.38 \pm 0.12$ & 2 & $0.41 \pm 0.11$ & 2 & -0.03 \\
\hline$[\mathrm{Al} \mathrm{I} / \mathrm{Fe} \mathrm{I}]$ & $0.72 \pm 0.20$ & 2 & $0.31 \pm 0.08$ & 2 & +0.41 \\
\hline$[\mathrm{Ca} \mathrm{I} / \mathrm{Fe} \mathrm{I}]$ & $0.36 \pm 0.07$ & 9 & $0.32 \pm 0.04$ & 8 & +0.04 \\
\hline$[\mathrm{Ti} \mathrm{I} / \mathrm{Fe} \mathrm{I}]$ & $0.34 \pm 0.08$ & 4 & $0.29 \pm 0.07$ & 4 & +0.05 \\
\hline$[\mathrm{Ti} \mathrm{II} / \mathrm{Fe} \mathrm{I}]$ & $0.27 \pm 0.09$ & 2 & $0.34 \pm 0.08$ & 2 & -0.07 \\
\hline [Ti II/Fe II] & $0.12 \pm 0.15$ & 2 & $0.28 \pm 0.13$ & 2 & -0.16 \\
\hline$[\mathrm{Cr} \mathrm{I} / \mathrm{Fe} \mathrm{I}]$ & $-0.13 \pm 0.06$ & 2 & $-0.14 \pm 0.07$ & 2 & +0.01 \\
\hline$[\mathrm{Mn} \mathrm{I} / \mathrm{Fe} \mathrm{I}]$ & $-0.16 \pm 0.12$ & 3 & $-0.22 \pm 0.14$ & 3 & +0.06 \\
\hline$[\mathrm{Ni} \mathrm{I} / \mathrm{Fe} \mathrm{I}]$ & $0.02 \pm 0.09$ & 2 & $0.01 \pm 0.08$ & 2 & +0.01 \\
\hline$[\mathrm{Cu} \mathrm{I} / \mathrm{Fe} \mathrm{I}]$ & $-0.63 \pm 0.20$ & 1 & $-0.34 \pm 0.10$ & 1 & -0.29 \\
\hline$[\mathrm{Zn} \mathrm{I} / \mathrm{Fe} \mathrm{I}]$ & $0.27 \pm 0.15$ & 1 & $0.06 \pm 0.10$ & 1 & +0.21 \\
\hline [Y II/Fe I] & $-0.13 \pm 0.15$ & 2 & $-0.04 \pm 0.09$ & 2 & -0.09 \\
\hline [Y II/Fe II] & $-0.28 \pm 0.17$ & 2 & $-0.10 \pm 0.13$ & 2 & -0.18 \\
\hline$[\mathrm{Ba} \mathrm{II} / \mathrm{Fe} \mathrm{I}]$ & $0.00 \pm 0.11$ & 3 & $0.16 \pm 0.08$ & 3 & -0.16 \\
\hline [Ba II/Fe II $]$ & $-0.15 \pm 0.16$ & 3 & $0.10 \pm 0.13$ & 3 & -0.25 \\
\hline$[\mathrm{Eu} \mathrm{II} / \mathrm{Fe} \mathrm{I}]$ & $<0.49$ & 1 & $0.30 \pm 0.15$ & 1 & - \\
\hline [Eu II/Fe II] & $<0.32$ & 1 & $0.36 \pm 0.18$ & 1 & - \\
\hline$[\mathrm{Ba} / \mathrm{Y}]$ & $0.13 \pm 0.18$ & - & $0.20 \pm 0.12$ & - & -0.07 \\
\hline$[\mathrm{Ba} / \mathrm{Eu}]$ & $>-0.49$ & - & $-0.14 \pm 0.17$ & - & - \\
\hline
\end{tabular}

Other high-resolution IL (and single star) analyses have found similar discrepancies between Fe I and Fe II (e.g., McWilliam \& Bernstein 2008, Colucci et al. 2009, 2012, 2014, Sakari et al. 2015, 2016). Such differences may reflect the weakness and paucity of Fe II lines in IL spectra. McWilliam \& Bernstein (2008) also suggest that offsets between Fe I and Fe II could occur as a result of incorrect modelling of the underlying stellar population; they list two possible sources of error: 1) a mismatch between the $[\alpha / \mathrm{Fe}]$ ratio of the cluster and the isochrone and 2) an incorrect number of bright asymptotic giant branch or tip of the RGB stars in the adopted model. Both possibilities were confirmed to have different effects on the IL $[\mathrm{Fe} \mathrm{I/H}]$ and $[\mathrm{Fe} \mathrm{II} / \mathrm{H}]$ ratios by Sakari et al. (2014), based on tests with 47 Tuc and other clusters. Non-LTE (NLTE) effects can also lead to lower Fe I abundances in LTE analyses (e.g., Lind et al. 2012; Amarsi et al. 2016); however, these effects are not very strong at G1's metallicity. Even if the underlying stellar population in G1 has not been perfectly modelled, the $[\mathrm{X} / \mathrm{Fe}]$ ratios are less sensitive to these effects than $[\mathrm{X} / \mathrm{H}]$ ratios (Sakari et al. 2014, though see Section 3.3 .

\subsubsection{Carbon}

The $\mathrm{C}$ abundance is difficult to ascertain from this spectrum, since the molecular lines are relatively weak and blended at this spectral resolution. Upper limits on $[\mathrm{C} / \mathrm{Fe}]$ were determined from $\mathrm{C}_{2}$ features at 5135, 5165, 5585, and $5635 \AA$. The upper limit of $[\mathrm{C} / \mathrm{Fe}]<0.17$ suggests that the cluster is not $\mathrm{C}$-enhanced. This lower value is consistent with expected values from tip of the RGB stars and with other IL analyses (e.g., Schiavon et al. 2013; Sakari et al. 2016).

\subsubsection{Sodium and Aluminum}

The sodium abundances were derived from two sets of $\mathrm{Na}$ I doublets: the lines at 5682 and $5688 \AA$ and those at 6154 and $6160 \AA$. Aluminum was derived from the 6696 and $6698 \AA$ lines. The syntheses for these features are shown in Figures 7 and 8 the latter figure also shows a comparison with 47 Tuc. Solar ratios of $[\mathrm{Na} / \mathrm{Fe}]=0$ and $[\mathrm{Al} / \mathrm{Fe}]=0$ are also shown, demonstrating that the cluster is enhanced in $\mathrm{Na}$ and $\mathrm{Al}$. Table 3 shows that these lines lead to $[\mathrm{Na} / \mathrm{Fe}]$ and $[\mathrm{Al} / \mathrm{Fe}]$ ratios in $\mathrm{G} 1$ that are about 0.2 and 0.4 dex higher, respectively, than 47 Tuc (see Section 4.1.1.

The two sets of Na lines are known to have NLTE corrections: for a typical RGB star in G1, the INSPECT database 10 (Lind et al. 2011) indicates that the corrections would be negative (up to $\sim-0.2$ dex for the $5682 / 5688 \AA$ lines). Similar corrections would also be needed in 47 Tuc. The metallicity difference between the two clusters could lead to different NLTE corrections; however, the INSPECT database indicates that the difference between NLTE corrections is not significant for $[\mathrm{Fe} / \mathrm{H}]=-1$ versus -0.7 . This indicates that G1's higher $[\mathrm{Na} / \mathrm{Fe}]$ cannot be explained solely with NLTE. Note that these NLTE corrections are not applied here. The implications of these $\mathrm{Na}$ and $\mathrm{Al}$ abundances will be discussed further in Section 4

10 http://inspect.coolstars19.com/ 

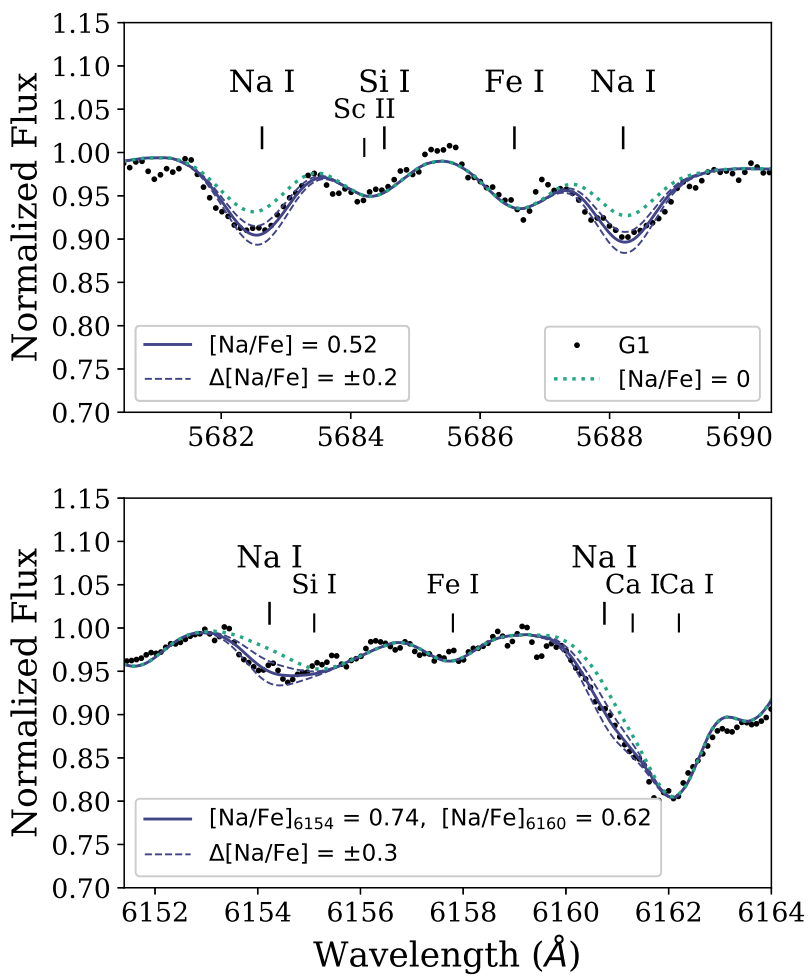

Figure 7. Syntheses of the $5682 / 5688 \AA$ (top) and $6154 / 6160$ A (bottom) Na I doublets. The black points show the G1 spectrum. The solid lines show the best-fitting syntheses, while the dashed lines show uncertainties of 0.2 and 0.3 , respectively. The dotted green line shows a solar $[\mathrm{Na} / \mathrm{Fe}]$ ratio; neither set of doublets is consistent with a solar $[\mathrm{Na} / \mathrm{Fe}]$ ratio.

\subsubsection{The $\alpha$ elements}

$\mathrm{Mg}, \mathrm{Ca}$, and $\mathrm{Ti}$ abundances were determined from a variety of spectral lines. In the case of $\mathrm{Mg}$, lines at 5528 and $5711 \AA$ were used-note that the $5528 \AA$ line is rather strong in G1 and 47 Tuc, while the $5711 \AA$ is barely detectable in G1. Both $\mathrm{Mg}$ lines in G1 yield a supersolar $[\mathrm{Mg} / \mathrm{Fe}]$ ratio (see Figure 9). Nine Ca lines were measured, spanning a broad range in wavelength; all indicate that $[\mathrm{Ca} / \mathrm{Fe}]$ is supersolar. Finally, four Ti I and two Ti II lines were measured, indicating elevated $[\mathrm{Ti} / \mathrm{Fe}]$. Note that the [Ti II/Fe II] ratio is lower than the [Ti II/Fe I] ratio, which may indicate a problem with the derived $[\mathrm{Fe}$ II/H] (see Section 3.2.1). Lower [Ti II/Fe II] ratios are not necessarily unusual; from IL observations, Colucci et al. (2017) found lower $[\mathrm{Ti} \mathrm{II} / \mathrm{Fe}]$ ratios than $[\mathrm{Ti} \mathrm{I} / \mathrm{Fe}$ ] for several Milky Way GCs. This offset between Ti I and Ti II may indicate that the weaker, bluer Ti II lines may be less reliable than the Ti I lines. Ultimately, G1 appears to be $\alpha$-enhanced, similar to 47 Tuc and the majority of the Milky Way and M31 GCs - this will be discussed in more detail in Section 4.2 .
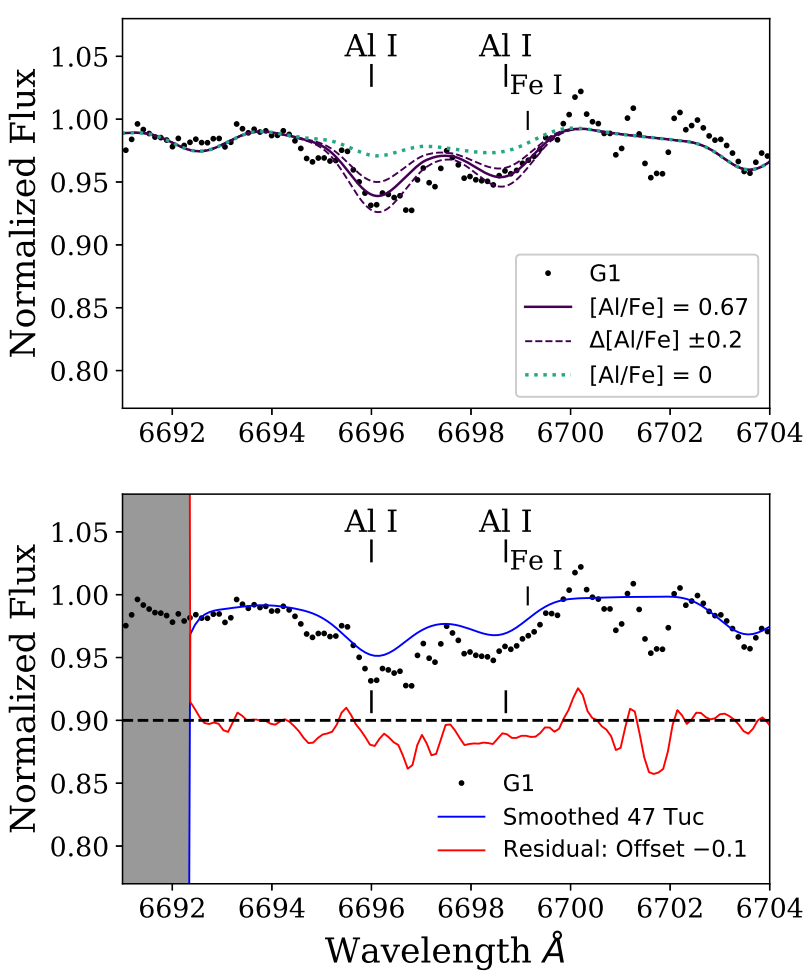

Figure 8. The 6696 and $6698 \AA \mathrm{Al}$ I lines in G1. The black points show the G1 spectrum. Top: Spectrum syntheses of the Al lines. The solid line shows the best-fitting syntheses, while the dashed lines show uncertainties of 0.2 and 0.3 , respectively. The dotted green line shows a solar $[\mathrm{Al} / \mathrm{Fe}]$ ratio; neither line is consistent with a solar $[\mathrm{Al} / \mathrm{Fe}]$ ratio. Bottom: A comparison with the 47 Tuc spectrum (solid blue line), which has been smoothed to G1's velocity dispersion. The residual is shown below the spectra, offset by -0.1 dex. As in Figure 1 the shading shows a bad region of the 47 Tuc spectrum.

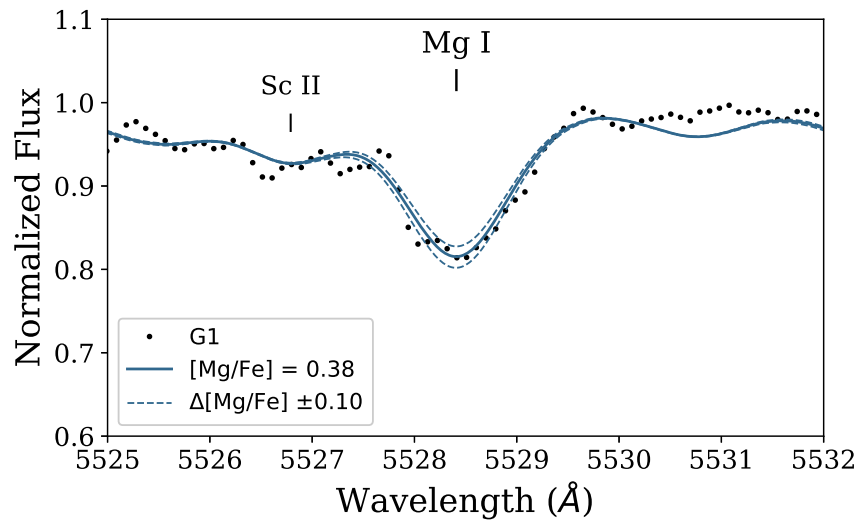

Figure 9. Syntheses of the $5528 \AA$ (left) and $5711 \AA$ (right) Mg I lines. The black points show the G1 spectrum. The solid line shows the best-fitting syntheses, while the dashed lines show uncertainties. 


\subsubsection{Iron-peak elements, $\mathrm{Cu}$, and $\mathrm{Zn}$}

Abundances of the iron-peak elements $\mathrm{Cr}, \mathrm{Mn}$, and $\mathrm{Ni}$ were determined from an assortment of spectral lines; in the case of Mn, HFS components were also included. $\mathrm{Cu}$ was determined from the $5782 \AA$ line (assuming a solar isotopic ratio; Asplund et al. 2009) with the HFS components from the Kurucz database 11 Note that the stronger 5105 $\AA \mathrm{Cu}$ I line was located in a lower $\mathrm{S} / \mathrm{N}$ region of the $\mathrm{G} 1$ spectrum, and was not included. Zn was determined from the 4810 A line.

$\mathrm{Cr}, \mathrm{Mn}$, and $\mathrm{Ni}$ are considered to be standard iron-peak elements, while $\mathrm{Cu}$ and $\mathrm{Zn}$ are though to form via weak $s$ processing (Bisterzo et al. 2004; Pignatari et al. 2010). Ultimately, G1's $[\mathrm{Ni} / \mathrm{Fe}]$ ratio is solar, $[\mathrm{Cr} / \mathrm{Fe}]$ and $[\mathrm{Mn} / \mathrm{Fe}]$ are slightly subsolar, $[\mathrm{Cu} / \mathrm{Fe}]$ is significantly subsolar, and $[\mathrm{Zn} / \mathrm{Fe}]$ is slightly elevated. These trends are generally similar to the results in 47 Tuc. Although $[\mathrm{Cu} / \mathrm{Fe}]$ is lower in G1, this is consistent with standard Milky Way chemical evolution at $[\mathrm{Fe} / \mathrm{H}] \sim-1$ versus -0.8 (see McWilliam et al. 2013). The high [Zn/Fe] in G1 agrees with typical Milky Way field stars within its errors (Bisterzo et al. 2004).

\subsubsection{Neutron capture elements}

Two Y II lines, at 5087 and $5200 \AA$, were used to determine $[\mathrm{Y} / \mathrm{Fe}]$, while three Ba II lines, at 5853, 6141, and $6496 \AA$, were used to determine [Ba/Fe]. All three of the Ba lines have isotopic splitting; a solar isotopic ratio was assumed Asplund et al. 2009). An upper limit in [Eu/Fe] for G1 was determined from the Eu II $6645 \AA$ line, including isotopic components and a Solar isotopic ratio (Asplund et al. 2009). The resulting G1 abundances show that $[\mathrm{Y} / \mathrm{Fe}]$ is slightly subsolar, $[\mathrm{Ba} / \mathrm{Fe}]$ is approximately solar (depending on whether Fe I or Fe II is used), and $[\mathrm{Eu} / \mathrm{Fe}]$ is moderately enhanced at most.

$\mathrm{Y}$ and $\mathrm{Ba}$ are mainly created by the slow $\left(s^{-}\right)$neutroncapture process, while $\mathrm{Eu}$ is mainly created by the rapid neutron-capture $(r-)$ process (Burris et al. 2000). The slightly elevated $[\mathrm{Ba} / \mathrm{Y}]$ ratio hints at a small excess of heavier neutron-capture elements; this small excess is also found in 47 Tuc, and is consistent with the general Milky Way trend. G1's $[\mathrm{Ba} / \mathrm{Eu}]$ ratio indicates that it is consistent with being dominated by $r$-process material, similar to 47 Tuc, though it may have received some small contributions from the $s$-process. The implications of the neutron-capture abundances in G1 will be discussed further in Section 4.1.3.

\subsubsection{Tuc: Comparison with Literature Abundances}

Previous IL abundances for 47 Tuc have been derived by McWilliam \& Bernstein (2008), Sakari et al. (2013, 2014), Colucci et al. (2017), and Larsen et al. (2017). This paper

11 http://kurucz.harvard.edu/linelists.html has presented a new set of IL abundances for 47 Tuc, using only the stronger lines that are detectable in G1. Offsets between the 47 Tuc abundances in this analysis versus the literature can provide insight into systematic offsets that could be present in the G1 abundances as well. These abundance offsets are shown in Figure 10

When comparing the results from this paper to these analyses from the literature, an important caveat is that McWilliam \& Bernstein (2008), Sakari et al. (2013, 2014), and Colucci et al. (2017) have all analysed the same 47 Tuc spectrum that is used in this paper, but with different techniques. Larsen et al. (2017) obtained a separate 47 Tuc spectrum. McWilliam \& Bernstein (2008), Sakari et al. (2013, 2014), and Larsen et al. (2017) used $H S T$ photometry to model the underlying populationsthis is only possible for nearby GCs whose stars can be resolved down to the main sequence. Colucci et al. (2017) used theoretical isochrones to model the underlying populations, similar to the analysis here. For the abundance analysis, McWilliam \& Bernstein (2008) performed an equivalent width (EW) analysis, Sakari et al. (2013, 2014) and Colucci et al. (2017) used a mixture of EWs and spectrum syntheses, and Larsen et al. (2017) used fullspectrum fitting with synthetic spectra. The choice of spectral lines and atomic data also vary between these papers. Although the analysis in this paper uses the same spectrum as McWilliam \& Bernstein (2008) and the subsequent analyses, this paper only uses the spectral lines that are detectable in G1.

Figure 10 shows that most of the points fall within the grey bar, which indicates the typical uncertainty for the $[\mathrm{X} / \mathrm{Fe}]$ ratios in this paper. There are likely to be systematic errors between the analyses as a result of the differences in modeling the underlying stellar populations (see, e.g., Sakari et al. 2014). One major difference is that, because of the requirement to only use lines that are detectable in G1, this analysis uses stronger lines than McWilliam \& Bernstein (2008), Sakari et al. (2013, 2014), or Colucci et al. (2017). A few of the key outliers Figure 10 are discussed below. The high-precision, differential abundances of individual 47 Tuc stars from Koch \& McWilliam (2008) are also included in this discussion.

Fe I: Although the $[\mathrm{Fe} \mathrm{I} / \mathrm{H}]$ ratio derived in this paper is generally in agreement with the literature analyses, Colucci et al. (2017) find a higher $[\mathrm{Fe} / \mathrm{H}]$, by $0.11 \mathrm{dex}$ $([\mathrm{Fe} / \mathrm{H}]=-0.65)$. This may be due to the line selection, since stronger lines are utilized in this analysis; however, 12 of the 25 lines in this analysis are also included in Colucci et al. (2017). With a similar line selection as Colucci et al., Sakari (2014) found [Fe I/H] $=-0.74 \pm 0.03$ with theoretical isochrones, which is in better agreement with the $[\mathrm{Fe} / \mathrm{H}]$ derived here.

In a differential analysis of nine individual stars in 47 Tuc, Koch \& McWilliam (2008) found a metallicity of $[\mathrm{Fe} \mathrm{I} / \mathrm{H}]=-0.76$, with a random error of 0.01 dex and a systematic error of 0.04 dex. The result from this paper is in excellent agreement with this high-quality values from individual stars.

Na: Colucci et al. (2017) find a Na abundance of 


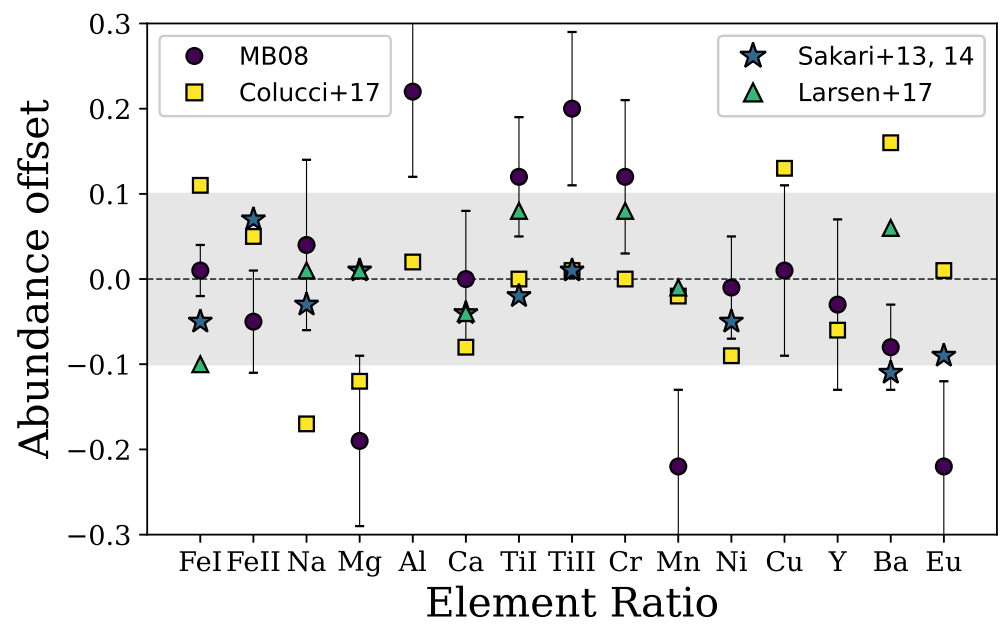

Figure 10. Abundance ratio offsets for 47 Tuc (literature - this paper). For Fe I and Fe II, $[\mathrm{Fe} / \mathrm{H}]$ ratios are compared. For all other elements, $[\mathrm{X} / \mathrm{Fe}]$ ratios are compared; Fe II ratios are used for $[\mathrm{X} / \mathrm{Fe}]$ ratios of singly ionized species. The literature data from McWilliam \& Bernstein (2008, purple circles), Sakari et al. (2013, 2014, blue stars), Colucci et al. (2017, yellow squares), and Larsen et al. (2017, green triangles) are shown. The grey bar shows a range of \pm 0.1 dex, which is a typical uncertainty for the [X/Fe] ratios in this paper. Representative error bars are shown for the McWilliam \& Bernstein (2008) points only.

$[\mathrm{Na} / \mathrm{Fe}]=0.24 \pm 0.08$, which is 0.14 dex lower than the value in this paper. The $\log \epsilon$ abundances from Colucci et al. are very similar to the values in this paper; instead, this offset is likely due to the way the $[\mathrm{Na} / \mathrm{Fe}]$ is calculated. Colucci et al. (2017) perform a differential analysis, relative to the solar abundance derived from the same lines. A differential analysis is not performed here, since the 5682 and $5688 \AA$ lines are prohibitively strong in the solar spectrum (Sakari et al. 2013). With the Asplund et al. (2009) solar value for Na, Colucci et al.'s $[\mathrm{Na} / \mathrm{Fe}]$ ratio is in closer agreement with the value derived here.

From individual stars, Koch \& McWilliam (2008) found a mean $[\mathrm{Na} / \mathrm{Fe}]=0.22 \pm 0.03$ dex. Although the IL $[\mathrm{Na} / \mathrm{Fe}]$ is higher than this value from individual stars, this is likely due to the presence of Na-enhanced stars within the cluster (see discussions in Sakari et al. 2013, Colucci et al. 2017, and Section 4.1.1).

Mg: McWilliam \& Bernstein (2008) and Colucci et al. (2017) both find a lower $[\mathrm{Mg} / \mathrm{Fe}]$ ratio for 47 Tuc.

Sakari et al. (2013) demonstrated that this may be due to an issue with the atomic data for the $7387 \AA$ line, which is the only $\mathrm{Mg}$ I line used by McWilliam \& Bernstein (2008) and one of three lines used by Colucci et al. (2017). The value derived in this paper is in excellent agreement with the mean value for the individual stars analyzed by Koch \& McWilliam (2008), $[\mathrm{Mg} / \mathrm{Fe}]=+0.46 \pm 0.05$.

Al: The abundances derived here are in agreement with Colucci et al. (2017), who used spectrum syntheses. However, with EW analyses McWilliam \& Bernstein (2008) derive a higher $[\mathrm{Al} / \mathrm{Fe}]$ of +0.53 for 47 Tuc. The discrepancy with McWilliam \& Bernstein (2008) is likely due to differences in the atomic data used for the 6696 and $6698 \AA$ lines. Adopting the $\log g f$ values from McWilliam \& Bernstein (2008) would increase the 47 Tuc (and G1) $[\mathrm{Al} / \mathrm{Fe}]$ ratios. From individual stars,
Koch \& McWilliam (2008) find an average $[\mathrm{Al} / \mathrm{Fe}]=+$ $0.45 \pm 0.06$, which is higher than the value derived in this paper.

Ti: McWilliam \& Bernstein (2008) find higher Ti I and $\mathrm{Ti}$ II ratios than those in this analysis. However, there is only one line in common for each ratio. This offset may therefore reflect uncertainties in atomic data or their EW measurements.

Cr and Mn: McWilliam \& Bernstein (2008) also find higher $[\mathrm{Cr} / \mathrm{Fe}]$ and lower $[\mathrm{Mn} / \mathrm{Fe}]$ ratios, compared to this analysis. Again, these offsets may be due to issues with measuring EWs, or they could reflect systematic differences in spectral lines (no Cr lines are similar, while only one $\mathrm{Mn}$ line is in common). In particular, differences in NLTE corrections between the various lines could lead to offsets in the final $[\mathrm{Cr} / \mathrm{Fe}]$ and $[\mathrm{Mn} / \mathrm{Fe}]$ ratios.

Cu: Colucci et al. (2017) find a higher, solar $[\mathrm{Cu} / \mathrm{Fe}]$ ratio for 47 Tuc. This discrepancy seems to caused by their inclusion of the $5105 \AA$ line. When only the $5782 \AA$ is considered, the ratios are in agreement.

Ba: McWilliam \& Bernstein (2008) and Sakari et al. (2014) found lower $[\mathrm{Ba} / \mathrm{Fe}]$ ratios than this analysis, which may be a result of using EWs rather than syntheses. On the other hand, Colucci et al. (2017) find a higher [Ba/Fe] ratio than the value in this paper, possibly as a result of different adopted isotopic ratios.

Eu: McWilliam \& Bernstein (2008) also find a lower $[\mathrm{Eu} / \mathrm{Fe}]$ ratio than the analysis in this paper; however, this may be because they used an EW rather than spectrum synthesis to derive the abundance (see the discussion in Sakari et al. 2013).

Most of the offsets in Figure 10 can therefore be explained with differences in line selection (leading to offsets because of, e.g., uncertain atomic data or solar ratios) or line measurement techniques (EWs versus spectrum syntheses). 
This comparison between the 47 Tuc analyses demonstrates that, in general, the usage of stronger spectral lines has not led to obvious systematic offsets in the $[\mathrm{X} / \mathrm{Fe}]$ ratios. This result is encouraging for the IL abundances of G1, whose detectable lines are similarly strong. The offsets in comparison with the literature abundances will be also present in the abundances of G1.

\subsection{Systematic Errors}

There are many systematic effects that can alter the derived IL abundance ratios. A full systematic error exploration is beyond the scope of this paper; a more detailed discussion of, e.g., the effects of AGB models, HB morphology, and isochrone binning is presented in Sakari et al. (2014). However, given the comparisons with 47 Tuc that are present throughout this paper, it is worth briefly discussing three specific sources of uncertainty in the isochrone modeling that were identified by McWilliam \& Bernstein (2008) as being particularly problematic for 47 Tuc: $M$ giants, mass segregation, and AGB bump stars. Cool $\mathrm{M}$ giants have significant $\mathrm{TiO}$ absorption that can affect IL spectral features, particularly at red wavelengths. 47 Tuc is known to have two M giants in its core; however, McWilliam \& Bernstein (2008) found that these two $\mathrm{M}$ giants have a negligible effect on the IL spectrum, causing only 0.02 and 0.01 dex offsets in $[\mathrm{Fe} \mathrm{I} / \mathrm{H}]$ and $[\mathrm{Fe} \mathrm{II} / \mathrm{H}]$, respectively. $\mathrm{M}$ giants become increasingly more prevalent in metal-rich clusters; since G1 is more metal-poor than 47 Tuc, it is unlikely to have a significant population of $\mathrm{M}$ giants (though a forthcoming paper will discuss how an iron spread could lead to $M$ giants in G1). The core region of 47 Tuc (which was covered in the IL spectrum used in this paper) is also known to suffer from mass segregation, which removes the lowest mass cluster stars. Sakari et al. (2014) found that adopting a low-mass cutoff did not have a significant effect on most abundance ratios. Finally, McWilliam \& Bernstein (2008) found that the BaSTI isochrones underpredicted the number of AGB bump stars in the 47 Tuc core. An incorrect number of AGB bump stars can have a significant effect on the derived abundances, since AGB stars are so bright. McWilliam \& Bernstein (2008) found that not including the extra AGB stars led to higher [Fe I/H] and $[\mathrm{Fe} \mathrm{II} / \mathrm{H}]$ ratios by $\sim 0.1$ and 0.15 dex, respectively. The exact abundance offsets will depend on the precise modeling of the AGB, the adopted cluster age, the spectral lines used, etc. The exact numbers of AGB bump stars may also vary from cluster to cluster. Such factor are difficult to model for G1.

In addition to these specific effects, there are two sources of systematic errors that are especially pertinent to G1: 1) the systematic errors that occur due to uncertainties in identifying an appropriate isochrone (this section) and 2 ) offsets that occur as a result of undetected abundance spreads within the cluster. The second source of uncertainty will be addressed in a forthcoming paper (Sakari et al., in prep.), while the first type is quantified below. Table 4 shows the abundance ratio uncertainties that occur when the isochrone age is changed by \pm 4 Gyr, the metallicity by
Table 4. Systematic uncertainties based on uncertainties in isochrone properties.

\begin{tabular}{lccccc}
\hline & \multicolumn{2}{c}{$\Delta$ Age $(\mathrm{Gyr})$} & \multicolumn{2}{c}{$\Delta[\mathrm{Fe} / \mathrm{H}]$} & $\Delta[\alpha / \mathrm{Fe}]$ \\
& -4 & +4 & -0.3 & +0.3 & -0.4 \\
\hline$\Delta[\mathrm{Fe} \mathrm{I} / \mathrm{H}]$ & +0.08 & -0.04 & +0.05 & 0.0 & -0.03 \\
$\Delta[\mathrm{Fe} \mathrm{II} / \mathrm{H}]$ & -0.01 & 0.0 & -0.16 & +0.23 & +0.03 \\
$\Delta[\mathrm{Na} / \mathrm{Fe}]$ & -0.02 & 0.0 & +0.03 & -0.07 & 0.0 \\
$\Delta[\mathrm{Mg} / \mathrm{Fe}]$ & 0.0 & 0.0 & +0.01 & -0.02 & +0.01 \\
$\Delta[\mathrm{Al} / \mathrm{Fe}]$ & -0.03 & +0.01 & +0.03 & -0.06 & 0.0 \\
$\Delta[\mathrm{Ca} / \mathrm{Fe}]$ & +0.01 & -0.01 & +0.06 & -0.08 & -0.01 \\
$\Delta[\mathrm{Ti} \mathrm{I} / \mathrm{Fe} \mathrm{I}]$ & +0.04 & -0.03 & +0.13 & -0.13 & -0.03 \\
$\Delta[\mathrm{Ti} \mathrm{II} / \mathrm{Fe} \mathrm{I}]$ & -0.03 & 0.0 & -0.11 & +0.14 & +0.03 \\
$\Delta[\mathrm{Ti} \mathrm{II} / \mathrm{Fe} \mathrm{II}]$ & +0.06 & -0.04 & +0.10 & -0.09 & -0.03 \\
$\Delta[\mathrm{Cr} / \mathrm{Fe}]$ & +0.03 & -0.02 & +0.08 & -0.11 & -0.02 \\
$\Delta[\mathrm{Mn} / \mathrm{Fe}]$ & +0.01 & 0.0 & +0.05 & -0.06 & -0.01 \\
$\Delta[\mathrm{Ni} / \mathrm{Fe}]$ & +0.01 & -0.01 & +0.01 & +0.02 & 0.0 \\
$\Delta[\mathrm{Cu} / \mathrm{Fe}]$ & -0.01 & +0.01 & +0.02 & 0.0 & 0.0 \\
$\Delta[\mathrm{Zn} / \mathrm{Fe}]$ & -0.02 & +0.01 & -0.08 & +0.10 & +0.03 \\
$\Delta[\mathrm{Y} \mathrm{II} / \mathrm{Fe} \mathrm{I}]$ & -0.02 & -0.01 & -0.11 & +0.11 & +0.03 \\
$\Delta[\mathrm{Y} \mathrm{II} / \mathrm{Fe} \mathrm{II}]$ & +0.07 & -0.05 & +0.10 & -0.12 & -0.03 \\
$\Delta[\mathrm{Ba} \mathrm{II} / \mathrm{Fe} \mathrm{I}]$ & -0.01 & 0.0 & -0.11 & +0.12 & +0.02 \\
$\Delta[\mathrm{Ba} \mathrm{II} / \mathrm{Fe} \mathrm{II}]$ & +0.08 & -0.04 & +0.10 & -0.11 & -0.04 \\
$\Delta[\mathrm{Eu} \mathrm{II} / \mathrm{Fe} \mathrm{I}]$ & -0.05 & +0.02 & -0.15 & +0.16 & +0.04 \\
$\Delta[\mathrm{Eu} \mathrm{II} / \mathrm{Fe} \mathrm{II}]$ & +0.04 & -0.04 & +0.06 & -0.07 & -0.02 \\
\hline & & & & &
\end{tabular}

\pm 0.3 dex, and the $[\alpha / \mathrm{Fe}]$ ratio by -0.4 . For neutral species all $[\mathrm{X} / \mathrm{Fe}]$ ratios utilize $\mathrm{Fe} \mathrm{I}$; for singly ionized species both $[\mathrm{X} / \mathrm{Fe} \mathrm{I}]$ and $[\mathrm{X} / \mathrm{Fe} \mathrm{II}]$ ratios are shown. For the purposes of this test, the $\mathrm{Eu}$ abundance was set to the upper limit value.

The results in Table 4 demonstrate that the $[\mathrm{Fe} \mathrm{I} / \mathrm{H}]$ ratio is relatively insensitive $(<0.1 \mathrm{dex})$ to the isochrone parameters ${ }^{12}$ while Fe II shows a strong sensitivity to isochrone metallicity. Most of the $[\mathrm{X} / \mathrm{Fe}]$ ratios are insensitive to age shifts of $4 \mathrm{Gyr}$, especially when the age is increased. Lowering the age to 6 Gyr does moderately affect many of the singly ionized species, but only when $[\mathrm{X} / \mathrm{Fe} \mathrm{II}]$ ratios are used. Changes in the isochrone metallicity have a much stronger effect on all abundance ratios other than $[\mathrm{Mg} / \mathrm{Fe}],[\mathrm{Ni} / \mathrm{Fe}]$, and $[\mathrm{Cu} / \mathrm{Fe}]$. In particular, the Ti, Zn, $\mathrm{Y}, \mathrm{Ba}$, and $\mathrm{Eu}$ abundance ratios can be affected by more than 0.1 dex. Changing the $[\alpha / \mathrm{Fe}]$ of the isochrone has a negligible effect on all abundance ratios.

From the results in Table 4 it is unclear whether $[\mathrm{X} / \mathrm{Fe} \mathrm{I}]$ or $[\mathrm{X} / \mathrm{Fe} \mathrm{II}]$ ratios are more robust for singly ionized species. In the case of $\mathrm{Ti}$ II, comparisons with Fe I lead to lower systematic errors for age shifts, while Fe II leads to lower offsets for $[\mathrm{Fe} / \mathrm{H}]$ shifts. For Y II and Ba II the differences between the Fe I and Fe II ratios are minimal for metallicity shifts, while Fe I is preferable for age shifts. Finally, for Eu II the Fe II ratios are more robust to age and metallicity shifts. Ultimately, there is a lot of complexity behind these abundance ratios, related to the specific spectral lines and their properties. However, because it is not immediately clear which Fe ionization state to use for

12 This insensitivity to the isochrone parameters makes it possible to use $[\mathrm{Fe} \mathrm{I} / \mathrm{H}]$ as a constraint on an appropriate isochrone age and metallicity, as in Figures 3 and 4 
singly ionized species, both are reported throughout this paper.

\section{DISCUSSION}

As mentioned in Section 1 G1 has long been identified as both a GC and a possible former NSC. Below these possibilities are discussed in the context of G1's detailed abundance ratios.

\subsection{G1 as a Globular Cluster}

Although G1 physically resembles a GC, there are many open questions as to whether it can truly be considered a GC and whether GC trends can be scaled up to G1's mass. This section discusses how G1's detailed abundances can shed light on this issue.

\subsubsection{Sodium and Aluminum enhancement}

One of the most striking results from this abundance analysis of $\mathrm{G} 1$ is the elevated $[\mathrm{Na} / \mathrm{Fe}]$ and $[\mathrm{Al} / \mathrm{Fe}]$ ratios, at +0.6 and +0.72 dex, respectively. A Na-O anticorrelation is a ubiquitous feature of classical Milky Way GCs, while many clusters have also found to exhibit a $\mathrm{Mg}-\mathrm{Al}$ anticorrelation (e.g., Carretta et al. 2009). Numerous IL studies of Milky Way and extragalactic GCs have found that many clusters have elevated IL $[\mathrm{Na} / \mathrm{Fe}]$ ratios and, in some cases, elevated $[\mathrm{Al} / \mathrm{Fe}]$ ratios, which may be signatures of multiple populations (e.g., Colucci et al. 2009, 2014, 2017, Sakari et al. 2013, 2015, 2016, Larsen et al. 2017, 2018a b). G1's elevated $[\mathrm{Na} / \mathrm{Fe}]$ and $[\mathrm{Al} / \mathrm{Fe}]$ seem to indicate that it too hosts Na- and Al-enhanced stars - the tests in Section 3.3 have demonstrated that elevated $\mathrm{Na}$ and $\mathrm{Al}$ cannot be caused by systematic errors in isochrone selection. Since elevated $[\mathrm{Na} / \mathrm{Fe}]$ and $[\mathrm{Al} / \mathrm{Fe}]$ are a unique signature of GCs, this finding indicates that G1 is intimately connected with the classical GCs.

G1's $[\mathrm{Na} / \mathrm{Fe}]$ and $[\mathrm{Al} / \mathrm{Fe}]$ ratios are also $\sim 0.2$ and $\sim 0.4$ dex higher than 47 Tuc's value, respectively. One possible interpretation of this enhancement is that G1 may possess a higher fraction of Na-enhanced stars (either I or E stars in the Carretta et al. 2010 framework) than 47 Tuc. G1 may have relatively more Na-enhanced stars as a result of its higher mass: Figure 11] shows the IL [Na/Fe] versus the cluster velocity dispersion, a proxy for cluster mass 13 for a sample of M31 GCs; 47 Tuc is also shown for comparison. This figure demonstrates that the higher mass, higher velocity dispersion clusters tend to have higher IL $[\mathrm{Na} / \mathrm{Fe}]$ ratios. G1's high $[\mathrm{Na} / \mathrm{Fe}]$ is very similar to B225, another massive cluster (Larsen et al. 2018b). This trend with mass is also reinforced by observations of Milky Way GCs, where more massive clusters seem to have relatively fewer of the "primordial" population stars

13 Note that the velocity dispersion reflects the current cluster mass, which may differ from its birth mass.
(Milone et al. 2017). Similar trends with mass (or proxies for mass) have also been previously detected in IL [Na/Fe] ratios (Colucci et al. 2014), $[\mathrm{Na} / \mathrm{O}]$ ratios (Sakari et al. 2016), and [N/Fe] ratios (Schiavon et al. 2013). A general interpretation of these trends with mass is that more massive clusters are able to retain more ejecta from the source of multiple populations, leading to an increased number of $\mathrm{Na}$ - and $\mathrm{N}$-enhanced stars (see the review by Bastian \& Lardo 2018). This result may extend to Al, indicating that G1 also has an intracluster spread in Al, similar to several Milky Way GCs (e.g., Carretta et al. 2009) 14

Follow-up spectroscopy further in the blue or in the infrared may better characterize the multiple populations in G1. Based on infrared IL spectra of other M31 GCs, Sakari et al. (2016) were able to obtain O abundances and found a significant trend in $[\mathrm{Na} / \mathrm{O}]$ versus cluster mass; however, $\mathrm{O}$ abundances cannot be determined from G1's optical spectrum. Similarly, a N abundance cannot be determined with the spectrum in this paper, meaning that G1 cannot yet be compared to the trend detected by Schiavon et al. (2013). Despite its high $[\mathrm{Al} / \mathrm{Fe}]$, it is worth noting that G1's $[\mathrm{Mg} / \mathrm{Fe}]$ agrees with $[\mathrm{Ca} / \mathrm{Fe}]$ and $[\mathrm{Ti} \mathrm{I} / \mathrm{Fe}]$, suggesting that there is not a significant $\mathrm{Mg}$ spread in the cluster. However, this is consistent with the individual stellar abundances in Milky Way GCs; Carretta et al. (2009) find that in many clusters a fairly large spread in $[\mathrm{Al} / \mathrm{Fe}]$ can be accompanied by a much smaller spread in $[\mathrm{Mg} / \mathrm{Fe}]$. Follow-up of stronger Al features may better characterize the nature of the multiplepopulations in G1.

To summarize, G1's elevated $[\mathrm{Na} / \mathrm{Fe}]$ and $[\mathrm{Al} / \mathrm{Fe}]$ suggest that it is intimately connected with GCs. Even if G1 is considered to be a NSC rather than a classical GC, it seems to have shared a common formation pathway with the classical GCs (see Section 4.2.1).

\subsubsection{Fe Spreads}

As mentioned throughout this paper, G1 has photometric evidence for intracluster Fe and He spreads (Meylan et al. 2001; Nardiello et al. 2019). The presence of an iron or helium spread does not automatically disqualify G1 as a GC. There are at least ten Milky Way GCs with broadened or bifurcated RGBs (the so-called "Type II" GCs; Milone et al. 2017); several of these GCs, notably $\omega$ Cen, have been confirmed to be iron-complex GCs (e.g., Johnson \& Pilachowski 2010). Milone et al. (2017) and Marino et al. (2019) also note that these Type II GCs can

14 Note that although 47 Tuc's stars are Al-enhanced, they may be consistent with standard Milky Way thick disk trends, with no intracluster spreads. Thygesen et al. (2014) find that an apparent Al spread could occur soley because of NLTE effects. Thygesen et al. (2016) further find no intracluster changes in $\mathrm{Mg}$ isotopes, suggesting that $\mathrm{Mg}-\mathrm{Al}$ cycling has not occurred in 47 Tuc. Mészáros et al. (2020) additionally find no evidence for an $[\mathrm{Al} / \mathrm{Fe}]$ spread within 47 Tuc. 


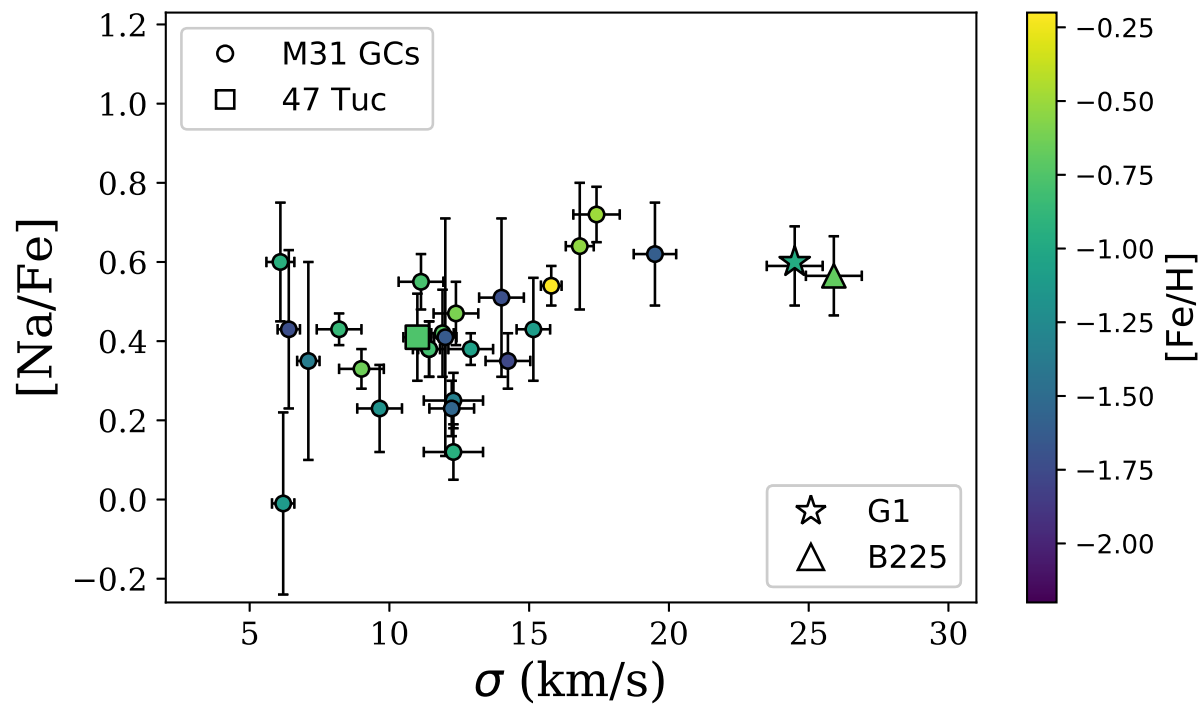

Figure 11. The IL $[\mathrm{Na} / \mathrm{Fe}]$ ratios in M31 GCs as a function of velocity dispersion. The star shows G1, the square shows 47 Tuc (this analysis), the circles show other M31 GCs (Colucci et al. 2014 and Sakari et al. 2015, 2016), and the triangle shows B225 Larsen et al. 2018b). The points are color-coded by cluster $[\mathrm{Fe} / \mathrm{H}]$.

show spreads in $\mathrm{He}, \mathrm{CNO}$, and $s$-process elements. A future paper (Sakari et al. in prep.) will investigate the effects of abundance spreads on the IL abundances, including whether such spreads could be detected in an IL spectrum. However, it is worth considering how G1's IL values agree with those of the Type-II GCs.

Amongst the population of Type-II Milky Way GCs, G1 falls on the massive and metal-rich end. Of the Type II clusters in Milone et al. (2017), only NGC 6388 has an average $[\mathrm{Fe} / \mathrm{H}]$ as high as G1 (Harris 1996: 2010 edition)the rest are predominantly more metal-poor. Marino et al. (2019) find that for Milky Way GCs the $\Delta[\mathrm{Fe} / \mathrm{H}]$ should increase with cluster mass; extrapolating the Milky Way results in their Figure 20 shows that G1 could have $\Delta[\mathrm{Fe} / \mathrm{H}] \gtrsim 0.4 \mathrm{dex}$, a spread that disagrees with the photometric analysis by Nardiello et al. (2019). One possibility for this disagreement is that the $H S T$ photometry of the outer regions misses part of G1's stellar population. Future follow-up photometry, e.g., in the crowded central regions, could help to characterize the metallicity distribution function in G1.

\subsubsection{Neutron Capture Abundances}

The abundances in Table 3 show that G1 has a solar $[\mathrm{Ba} / \mathrm{Fe}]$ ratio, slightly subsolar $[\mathrm{Y} / \mathrm{Fe}]$, and a moderate upper limit in $[\mathrm{Eu} / \mathrm{Fe}]$, indicating that it is not significantly enhanced in neutron capture elements. These values are similar to those of 47 Tuc and other classical Milky Way GCs at G1's metallicity. This lack of enhancement is distinct from $\omega$ Cen, whose intermediate metallicity stars have supersolar $[\mathrm{La} / \mathrm{Fe}]$ ratios
(Johnson \& Pilachowski 2010) 15 the stars in $\omega$ Cen show a clear rise in $[\mathrm{La} / \mathrm{Fe}]$ with increasing metallicity above $[\mathrm{Fe} / \mathrm{H}] \sim-1.7$ (Johnson \& Pilachowski 2010; Marino et al. 2011a). Although this increasing trend may flatten in the most metal-rich stars, most of $\omega$ Cen's metal-rich stars are still enhanced in La (Johnson \& Pilachowski 2010). The low IL $[\mathrm{Ba} / \mathrm{Fe}]$ for G1 suggests that G1's stars are not significantly enhanced in $\mathrm{Ba}$, and therefore that G1 has not experienced the same ongoing enrichment in neutroncapture elements (though see Sakari et al., in prep., for tests of abundance spreads).

G1's lower limit in $[\mathrm{Ba} / \mathrm{Eu}]$ shows that the cluster has experienced at least some small enrichment in $s$-process elements. Again, this level of $s$-process enrichment is consistent with the chemical evolution pattern of typical Milky Way field stars and GCs like 47 Tuc. However, G1 is again discrepant from $\omega$ Cen, which has supersolar enhancement in $[\mathrm{La} / \mathrm{Eu}]$. A high $[\mathrm{La} / \mathrm{Eu}]$ ratio indicates that the high $[\mathrm{La} / \mathrm{Fe}]$ ratios in $\omega$ Cen are the result of the $s$-process rather than the $r$-process. Since this analysis can only provide a lower limit in $[\mathrm{Ba} / \mathrm{Eu}]$ for $\mathrm{G} 1$, it is possible that the cluster could have had more contributions from the $s$ process, but this seems unlikely based on its solar $[\mathrm{Ba} / \mathrm{Fe}]$ ratio.

D'Orazi et al. (2011) also note that $\omega$ Cen's metal-rich stars have supersolar $[\mathrm{Ba} / \mathrm{Y}]$ ratios, which indicates an excess of lighter $s$-process elements like Y. They argue that the low $[\mathrm{Ba} / \mathrm{Y}]$ in $\omega$ Cen is a signature of extra contribu-

15 La is primarily an $s$-process element, like Ba Burris et al. 2000). Because its lines are weaker, La is often more desirable for individual stellar analyses; however, these weaker lines are not detectable in G1. 
tions from the higher mass AGB stars. Since G1's [Ba/Y] ratio is supersolar (again, in agreement with 47 Tuc), it seems that G1 does have a slight enhancement in lighter $s$-process elements. This $[\mathrm{Ba} / \mathrm{Y}]$ ratio will be discussed further in Section 4.2 .2

Altogether, G1's IL neutron-capture element ratios are similar to 47 Tuc and other classical GCs. Unlike $\omega$ Cen, G1's neutron capture abundances agree with those expected for a standard Milky Way cluster at $[\mathrm{Fe} / \mathrm{H}]=-1$. Of course, the stars in $\omega$ Cen have been studied individually, not via IL, which makes it easier to characterize the intracluster trends. The second paper in this series, Sakari et al. (in prep.), will examine the effects of intracluster abundance spreads on the IL spectrum.

\subsubsection{Comparisons with M31 GCs}

Compared with Milky Way GCs, G1 is unusual because it is is more massive and more metal-rich than typical Milky Way GCs. M31, however, contains multiple, massive, G1-like clusters. A handful of these GCs (B023, B158, and B225) have been photometrically identified as candidate iron-complex clusters, all of which were found to have $[\mathrm{Fe} / \mathrm{H}] \sim-0.9$ to -0.6 (Fuentes-Carrera et al. 2008). Colucci et al. (2014), Sakari et al. (2016), and Larsen et al. (2018b) have all conducted high-resolution IL spectroscopic analyses of B225, finding $[\mathrm{Fe} / \mathrm{H}] \sim-0.6$. Sakari et al. (2016) and Larsen et al. (2018b) further found minimal offsets between the optical and infrared $[\mathrm{Fe} / \mathrm{H}]$ ratios, placing constraints on the extent of an iron spread. Another massive M31 GC, B088, is more metalpoor $([\mathrm{Fe} / \mathrm{H}]=-1.7$; Sakari et al. 2016), similar to $\omega$ Cen; Sakari et al. (2016) suggested that variations in $[\mathrm{Mg} / \mathrm{Fe}]$ between the optical and infrared could indicate an iron spread within B088. There are several additional massive clusters that have not been studied in any detail. Ultimately, G1 is not unique in being a massive, moderately metal-poor star cluster with a possible iron spread. While it is the only one of these iron-complex clusters that is obviously in the outer halo (Mackev et al. 2019), Perina et al. (2012) argue that B023 and B225 are actually outer-halo clusters that are projected into the inner regions.

Other than its enhanced [Na/Fe], G1 seems chemically similar to other M31 GCs. Unlike the Milky Way GCs, which exhibit a bimodal $[\mathrm{Fe} / \mathrm{H}]$ distribution (e.g., Freeman \& Norris 1981), the M31 GCs don't have a clear $[\mathrm{Fe} / \mathrm{H}]$ bimodality (Caldwell et al. 2011). M31 also contains more metal-rich GCs than the Milky Way: the median $[\mathrm{Fe} / \mathrm{H}]$ of the Caldwell et al. (2011) sample is $[\mathrm{Fe} / \mathrm{H}]=-0.90 \pm 0.07$, compared to a median $[\mathrm{Fe} / \mathrm{H}]=-1.35 \pm 0.14$ in the Milky Way Harris 1996: 2010 edition). G1 therefore has a typical metallicity for an M31 GC. Although the outer halo GCs are, in general, more metal-poor than those in the inner regions (e.g., Caldwell et al. 2011), there are multiple metal-rich GCs that are projected into the outer-halo (e.g., Sakari et al. 2015). G1's $[\alpha / \mathrm{Fe}]$ enhancement is also in agreement with other M31 GCs at $[\mathrm{Fe} / \mathrm{H}] \sim-1$ in the inner and outer halo (see Figure 12).
G1's properties are therefore consistent with those of other M31 GCs, including those in the outer halo. Its mass-tolight ratio is also similar to other M31 GCs (Strader et al. 2009). If it were not for its high mass and high [Na/Fe], G1 would be considered a typical M31 GC based on its IL abundances. However, G1's high mass and its presence in the outer halo do raise some questions about where and how it formed. Many other open questions also remain about the origin of the outer halo and its GCs in general (e.g., McConnachie et al. 2018; Mackev et al. 2019). G1's relationship to the outer halo of M31 will be discussed further in Section 4.2 .3 .

\subsection{G1's Potential Origins in a Dwarf Galaxy}

Because of its mass and putative Fe spread, G1 has long been identified as the potential former NSC of a dwarf galaxy, like $\omega$ Cen. Under this paradigm, G1 would have assembled at the center of a satellite dwarf through GC mergers, in situ star formation, or a combination of the two processes (see Neumayer et al. 2020). As this dwarf galaxy fell into M31's gravitational potential, the outer field stars (and other GCs, if present) in the dwarf galaxy would have been stripped, creating stellar streams (see, e.g., simulations by Bekki \& Chiba 2004). Even if G1 was not a NSC, its location in the outer halo $\left(R_{\text {proj }}=34.7\right.$ kpc; Mackev et al. 2019) suggests that it may have originated as a GC in a dwarf galaxy. This section discusses G1's origins in light of its detailed abundances.

\subsubsection{Connections with Known Nuclear Star Clusters}

NSCs are found in many types of galaxies, from dwarf ellipticals to massive spirals like the Milky Way. The NSCs themselves have a wide range of properties, varying with host galaxy type, galaxy mass, NSC mass, etc. (see, e.g., Neumaver et al. 2020). This discussion focuses specifically on the NSCs that are associated with dwarf galaxies; however, it is worth noting that the properties of NSCs can still vary significantly, even in dwarf galaxies.

Past photometric results showed that NSCs in dwarf galaxies have similar colors as the Milky Way GCs which have extremely blue horizontal branches (including $\omega$ Cen), which suggests that dwarf galaxy NSCs and GCs have similar ages and metallicities (Georgiev et al. 2009). Ordenes-Briceño et al. (2018) similarly found dwarf nuclei to have colors consistent with metal-poor stellar populations. Using a variety of techniques, several mediumresolution IL spectroscopic studies of NSCs in dwarfs have found varying metallicities. Spengler et al. (2017) performed Lick index analyses on 12 confirmed or candidate dwarf elliptical NSCs in the Virgo galaxy cluster, finding ages $\sim 2-11 \mathrm{Gyr}$ and $[\mathrm{Fe} / \mathrm{H}] \sim-1.2$ to -0.15 . Kacharov et al. (2018) and Fahrion et al. (2020) used full spectrum fitting with different techniques to model the stellar populations in NSCs that are associated with a variety of galaxy types. For a single NSF in a dwarf elliptical/S0 galaxy NSC, Kacharov et al. (2018) found an age pf $\sim 0.5-3$ Gyr and $[\mathrm{Fe} / \mathrm{H}] \sim-0.4$ to -0.2 , depending on the 
fitting technique used. Fahrion et al. (2020) found the nuclei of two dwarf satellites of Cen A to be older ( $\sim 7$ Gyr) and more metal-poor $([\mathrm{Fe} / \mathrm{H}] \sim-1.8)$. At $[\mathrm{Fe} / \mathrm{H}]=-0.98$, G1 certainly falls within the observed metallicity ranges of NSCs. Though its age of 10 Gyr is older than most of the estimates for other NSCs, it is important to remember that these ages were derived with different techniques and that it is notoriously difficult to obtain ages from IL spectra (as discussed in Fahrion et al. 2020) 16

It is also worth considering whether G1 agrees with the trends from known NSCs. Amongst populations of NSCs in early and late-type galaxies, G1's velocity dispersion and mass-to-light ratio falls within the known ranges (e.g., Böker et al. 2004; Leigh et al. 2012). Spengler et al. (2017) and Sánchez-Janssen et al. (2019) both present empirical relationships between the mass of a NSC and the stellar mass of its host galaxy. For G1, with a mass of $\sim 10^{7.2} \mathrm{M}_{\odot}$ (Mevlan et al. 2001; Nardiello et al. 2019), these empirical trends would suggest that G1 originated in a galaxy with a stellar mass $M_{\star}=10^{8}-10^{10} \mathrm{M}_{\odot}$, i.e., about the mass of the Magellanic clouds Kim et al. 1998). Neumaver et al. (2020) also present a compilation of galaxy masses and NSC metallicities from the literature. This relation shows that G1's $[\mathrm{Fe} / \mathrm{H}]$ is consistent with origins in a galaxy with a stellar mass $\sim 10^{9} \mathrm{M}_{\odot}$-note that Neumaver et al. (2020) argue that NSC formation pathways change at this galaxy mass. They predict that NSCs associated with lower mass galaxies form primarily through GC mergers, while those associated with more massive galaxies form primarily through ongoing in situ star formation. G1's enhanced $[\mathrm{Na} / \mathrm{Fe}]$ indicates that, if it is a NSC, it has an intimate connection with GCs.

It is also worth noting that G1 does not appear to contain stars as metal-poor as M54, the NSC of the Sagittarius (Sgr) dwarf spheroidal (Mucciarelli et al. 2017). A lack of metal-poor stars could also suggest that if G1 is a NSC, then it originated in a galaxy more massive than Sgr, which is estimated to have had a total stellar mass $\sim 10^{8}$ $\mathrm{M}_{\odot}$ (Vasiliev \& Belokurov 2020) 17 More work should be done to understand whether G1 truly fits in with the population of NSCs.

\subsubsection{Clues from Chemical Abundances}

G1 shows enhanced $[\alpha / \mathrm{Fe}]$, based on its $[\mathrm{Mg} / \mathrm{Fe}],[\mathrm{Ca} / \mathrm{Fe}]$, and $[\mathrm{Ti} / \mathrm{Fe}]$ ratios. Figure 12 compares G1's IL $[\mathrm{Ca} / \mathrm{Fe}]$ vs. $[\mathrm{Fe} / \mathrm{H}]$ ratios to field stars in the Milky Way and GCs in the LMC and M31. Plots such as this are often used to unravel a galaxy's chemical evolution history, specifically the onset of Type Ia supernovae which leads to a downturn in $[\mathrm{Ca} / \mathrm{Fe}]$ with increasing $[\mathrm{Fe} / \mathrm{H}]$ (see, e.g.,

16 Note that Spengler et al. (2017) find older ages for many of their NSCs when photometric spectral energy distributions are used.

17 Note that current mass estimates of the Sgr stream are lower than than the total mass estimate from Vasiliev \& Belokurov $(2020)$ because the galaxy is being tidally disrupted.

Peñarrubia et al. (2011) find that as much as $40-50 \%$ of the initial stellar mass may have been lost.
Matteucci \& Brocato 1990; Tolstoy et al. 2009). Several papers have used the $[\alpha / \mathrm{Fe}]$ ratios to identify GCs that may have been accreted from low-mass dwarf galaxies. In particular, the three metal-poor GCs in Figure 12 with lower $[\mathrm{Ca} / \mathrm{Fe}]$ than the other M31 GCs (G002, B457, and PA-17) have been identified as candidate accreted GCs (Colucci et al. 2014; Sakari et al. 2015, 2016). G1's high $[\mathrm{Ca} / \mathrm{Fe}]$ at $[\mathrm{Fe} / \mathrm{H}]=-0.98$ indicates that it could not have originated in a very low-mass dwarf spheroidal.

McWilliam et al. (2013) note that $\mathrm{Cu}$ is another useful element for chemical tagging. In their analysis of three stars in the Sgr dwarf spheroidal, McWilliam et al. (2013) found lower $[\mathrm{Cu} / \mathrm{Fe}]$ compared to Milky Way field stars. However, the $[\mathrm{Cu} / \mathrm{O}]$ ratio fit the general trend with $[\mathrm{Fe} / \mathrm{H}]$ seen in field stars, consistent with metallicity-dependent $\mathrm{Cu}$ production by massive stars (e.g., Pignatari et al. 2010). G1's $[\mathrm{Cu} / \mathrm{Fe}]$ ratio, although low, is consistent with the $\mathrm{Cu}$ abundances of typical Milky Way field stars at $[\mathrm{Fe} / \mathrm{H}]=-1 ; 47$ Tuc's higher $[\mathrm{Cu} / \mathrm{Fe}]$ ratio, compared to G1, can be explained by normal chemical evolution. G1's $[\mathrm{Cu} / \mathrm{Fe}]$ ratio is therefore inconsistent with formation in a very low-mass galaxy.

In Sgr dwarf spheroidal stars, McWilliam et al. (2013) also found low $[\mathrm{Mg} / \mathrm{Ca}]$ ratios, which they attributed to a toplight IMF, i.e., a lack of the most massive stars, within Sgr. They argued that a top-light IMF could be a natural outcome in a low-mass system which might be unable to form the large giant molecular clouds that are needed to create the highest mass stars. G1's normal $[\mathrm{Mg} / \mathrm{Ca}]$ ratio requires no modifications to the IMF.

Finally, supersolar $[\mathrm{Ba} / \mathrm{Y}]$ (or $[\mathrm{La} / \mathrm{Y}]$ ) has also been identified as a chemical signature of stars that form in low-mass dwarf galaxies (e.g., Shetrone et al. 2003; Letarte et al. 2010; Sakari et al. 2011). One explanation for high [Ba/Y] ratios is that the $s$-process elements in dwarf galaxies are created in lower-metallicity AGB stars; as a result of their lower metallicity, these AGB stars have fewer seed nuclei, and can build up their $s$-process elements to higher proton numbers. G1 shows only a moderate enhancement in $[\mathrm{Ba} / \mathrm{Y}]$, which is again consistent with 47 Tuc and other Milky Way field stars and clusters. It therefore seems that G1's $s$-process enhancement did not come from AGB stars that were as metal-poor as those in very low-mass dwarf galaxies.

Altogether, G1's abundances are more consistent with origins in a fairly massive galaxy, perhaps one that was at least as massive as the Large Magellanic Cloud (LMC), which has a stellar mass $\sim 10^{9} \mathrm{M}_{\odot}$ ( Kim et al. 1998). It is worth noting that the presence of $s$-process elements requires contributions from AGB stars, while the elevated $[\alpha / \mathrm{Fe}]$ ratios rules out contributions from Type Ia supernovae. This places important constraints on the timescales for G1's formation: AGB star evolution requires timescales $\sim 1$ Gyr (e.g., Pietrinferni et al. 2004) while Type Ia supernovae start occurring on a similar timescale (Maoz et al. 2010). Future follow-up observations of, e.g., $\mathrm{Rb}$ and $\mathrm{Zr}$ could further constrain the mass of the AGB progenitors that created the $s$-process material in G1. 


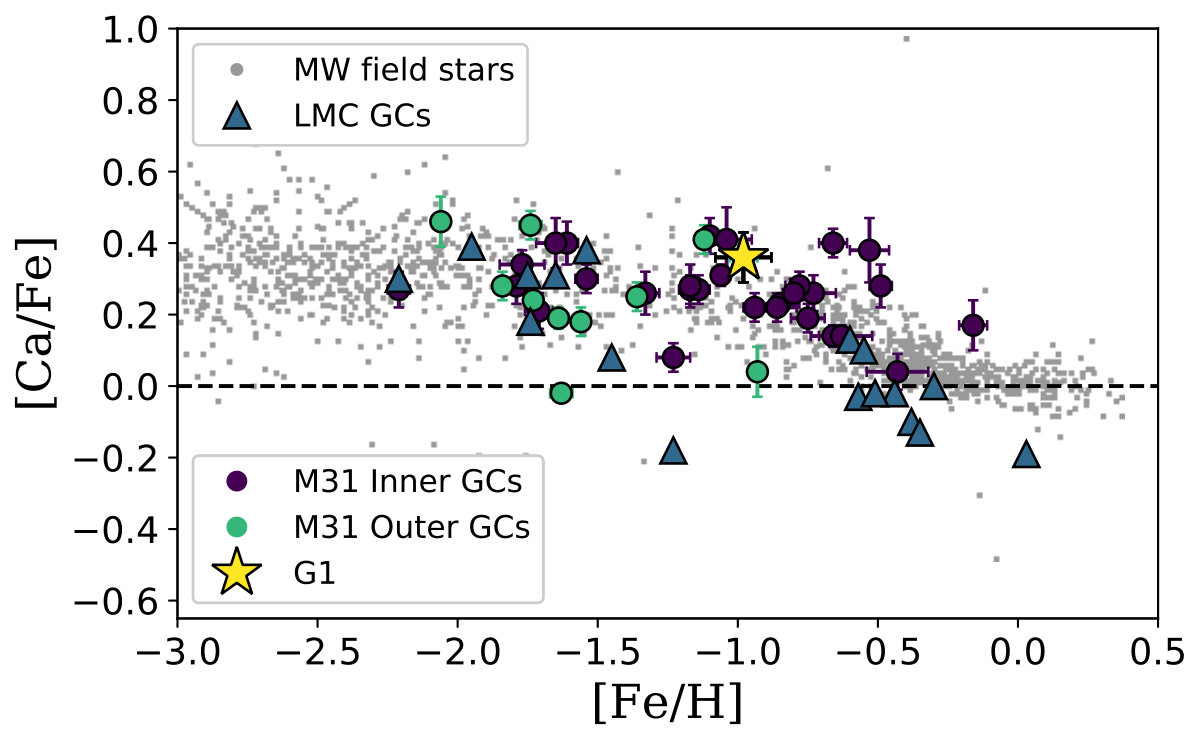

Figure 12. $[\mathrm{Ca} / \mathrm{Fe}]$ versus $[\mathrm{Fe} / \mathrm{H}]$ in $\mathrm{G} 1$ (yellow star) compared with Milky Way field stars (grey points; Venn et al. 2004; Reddy et al. 2006; Sakari et al. 2018), LMC GCs (IL and averages of individual stars; triangles, Johnson et al. 2006; Mucciarelli et al. 2008, 2010; Colucci et al. 2012; Sakari et al. 2017), and M31 inner and outer GCs (IL abundances; circles, Colucci et al. 2014; Sakari et al. 2015, 2016).

\subsubsection{Connections with M31's Outer Halo}

One of the intriguing characteristics of G1 is that though it has long been identified as a potential accreted cluster, it has no clear links with other GCs, intact dwarfs, or bright stellar streams. In projection, G1 does seem to lie near a group of GCs known as "Association 2"-however, G1's discrepant radial velocity suggests that it is not actually physically associated with those GCs (Velianoski et al. 2014). G1 is also projected close to a stellar over-density known as the "G1 clump" (so-named due to its proximity to G1; Ferguson et al. 2002), although these stars seem to be from M31's disk (Ferguson et al. 2005; Faria et al. 2007; Richardson et al. 2008). From spectra of individual stars in a region near G1 and the G1 clump, Reitzel et al. (2004) found that only a handful of stars had radial velocities consistent with G1, indicating that G1 likely does not have bright tidal tails. There is also no photometric evidence of stellar debris surrounding G1 (e.g, Mackey et al. 2019) 18 The question then arises: if G1 did originate in a dwarf galaxy, where is the remainder of that galaxy now?

One potential explanation for a lack of bright streams around G1 is that the host galaxy was accreted early on, so that any streams have since dissolved

18 Note that Mackev et al. (2019) used a "density percentile value" to quantify the density of stars surrounding outer halo GCs; they subsequently used this value to identify clusters that may be associated with faint substructure. Although G1 has a low density percentile value, indicating an absence of bright substructure, Mackey et al. (2019) classified G1 as a cluster that is associated with substructure, based on its possible classification as a NSC. (e.g., Johnston et al. 2008). Ibata et al. (2014) and Mackey et al. (2019) argue that early accretion can explain the lack of substructure in the most metal-poor outer halo stars and some of the GCs. However, outer halo stars with metallicities as high as G1 are primarily associated with substructure, particularly the Giant Stellar Stream to the south of M31 (Ibata et al. 2014). The lack of a "smooth" metal-rich component in the outer halo may indicate that a massive, metal-rich, LMC-like galaxy could not have been accreted very early on. This discrepancy may worsen if G1 was a NSC-Johnston et al. (2020) find evidence that NSCs in the Fornax cluster are generally more metal-poor and $\alpha$-rich than the field stars in their main galaxy. If G1's host contained stars more metal-rich than $[\mathrm{Fe} / \mathrm{H}]>-0.7$, it is not clear where those stars are now. Alternatively, a lack of nearby streams could indicate that the NSC was a dominant part of the galaxy's total mass. Sánchez-Janssen et al. (2019) find that in low-mass galaxies a NSC can comprise up to $\sim 50 \%$ of the galaxy's total stellar mass. Of course, comparisons with more isolated, intact nucleated dwarfs may not be appropriate, since G1's host galaxy may have been disturbed during its early evolution. Ultimately, deeper imaging of the vicinity around G1 could help assess the presence of stellar streams around the cluster.

Intuitively, G1's lack of associated GCs seems problematic, since it is unusual for massive galaxies to have only a single, massive, metal-rich GC; such a galaxy would likely have a very low specific frequency (e.g., Harris et al. 2013). However, if G1 was a NSC it may have no associated GCs. Based on observations of NSCs in the Virgo cluster, Sánchez-Janssen et al. (2019) find that though GCs or NSCs are similarly common in galaxies (i.e., the two ob- 
jects similar "occupation fractions"), the number of galaxies with both NSCs and GCs is slightly lower. In this scenario, G1's host galaxy may not have possessed any other GCs, which explains why none can be kinematically and chemically linked to G1 now 19 It is more unusual, however, that G1's progenitor would not have contained any metal-poor GCs, since most galaxies, especially dwarfs, have a significant population of metal-poor GCs (see, e.g., Brodie \& Strader 2006; several metal-poor LMC clusters are also visible in Figure 12). More modelling should be done to see if G1 can be kinematically linked to any other GCs or known stellar streams.

It is worth noting that other than its high [Na/Fe], G1 chemically resembles the classical M31 GCs, the Milky Way GCs (e.g., 47 Tuc), and the Milky Way disk stars. One simple explanation for this chemical similarity in the different environments is that G1 formed in M31 itself, rather than in a dwarf satellite. Its proximity to the G1 clump, material that is believed to have originated in M31's disk, further hints that G1 could have been born out of material from the disk. Based on observations of lower mass clusters in M31's disk, Johnson et al. (2017) argue that the high-mass limit of the cluster mass function depends on a galaxy's star formation rate surface density (i.e., the intensity of local star formation). The formation of a massive cluster like G1 through in situ star formation alone would require a very high rate of star formation. One could perhaps envision a scenario that would lead to the formation of a massive cluster in the disk, e.g., an interaction with a very high mass satellite galaxy-however, it is unclear how a massive cluster like G1 could be removed from the disk and brought into the outer halo.

Finally, it is worth noting that chemically G1 is also very similar to B225, the other massive cluster that has been studied at high spectral resolution, except that B225 is slightly more metal-rich (Colucci et al. 2014; Sakari et al. 2016; Larsen et al. 2018b). There are several additional unstudied, massive GCs in the inner regions, many of which appear to be similarly metal-rich. Any formation scenario for G1 should be able to explain the properties of these other GCs as well. Given that G1 seems to lack metal-poor stars (see the photometric limits on $\Delta[\mathrm{Fe} / \mathrm{H}]$ by Nardiello et al. 2019), a GC merger scenario would have to serendipitously involve GCs of roughly the same moderate metallicity. Such a scenario seems unlikely for all the massive, metal-rich GCs in M31's halo. Follow-up observations of these other clusters, specifically to characterize the intracluster iron spreads, would be useful for interpreting the results in G1.

Ultimately the abundances derived in this paper cannot shed light on whether G1 originated in a dwarf galaxy or not, though they do indicate that its birth site was a fairly massive galaxy, at least as massive as the LMC.

19 Note that G1 is kinematically and spatially close to the cluster G002-however, G002 is much more metal-poor $([\mathrm{Fe} / \mathrm{H}]=-1.63)$ and $\alpha$-poor $([\mathrm{Ca} / \mathrm{Fe}]=-0.02$; Colucci et al. 2014) than G1. It is difficult to imagine a scenario where the two could be associated without invoking complicated chemical evolution scenarios.

\section{CONCLUSION}

This paper has presented a high-resolution, IL spectroscopic abundance analysis of the massive M31 cluster, G1. A future paper will explore the effects of intracluster abundance spreads on the IL abundances. When a single age and metallicity is adopted for the entire cluster, G1 was found to be old, with an age of $10 \mathrm{Gyr}$, and moderately metal-poor, at $[\mathrm{Fe} / \mathrm{H}]=-0.98 \pm 0.05$. The cluster has $\alpha$, $[\mathrm{Cu} / \mathrm{Fe}],[\mathrm{Mg} / \mathrm{Ca}]$, Fe-peak and neutron-capture element abundance ratios that are typical for Milky Way and M31 GCs at $[\mathrm{Fe} / \mathrm{H}]=-1$. This comparison is strengthened by the similarity between G1 and the Milky Way cluster 47 Tuc. These abundances place constraints on G1's birth environment, suggesting that G1 formed in a galaxy that was at least as massive as the LMC.

G1 was also found to have elevated $\mathrm{Na}$ and $\mathrm{Al}$, with ratios $[\mathrm{Na} / \mathrm{Fe}]=+0.60$ and $[\mathrm{Al} / \mathrm{Fe}]=+0.72$, suggesting that it contains a high fraction of $\mathrm{Na}$ - and Al-enhanced (and, presumably, O-deficient) stars, a unique chemical signature of GCs. This result indicates that G1 shared a similar formation pathway as GCs. If G1 is a former NSC, it could have formed through in situ star formation, GC mergers, or a combination of the two, as long as the formation pathway led to enhanced $[\mathrm{Na} / \mathrm{Fe}]$ and $[\mathrm{Al} / \mathrm{Fe}]$. G1's $[\mathrm{Na} / \mathrm{Fe}]$ and $[\mathrm{Al} / \mathrm{Fe}]$ ratios are higher than the value for $47 \mathrm{Tuc}$; the $[\mathrm{Na} / \mathrm{Fe}]$ ratio agrees with previous IL observations of lower mass clusters that indicate a trend of increasing $[\mathrm{Na} / \mathrm{Fe}]$ with cluster mass. This trend may reflect that the most massive clusters possess larger relative amounts of Na-enhanced stars. This result has important consequences for models that seek to explain the formation of multiple populations in GCs.

One simple explanation for G1's abundance pattern is that it formed in a very massive giant molecular cloud, and experienced some amount of prolonged star formation and self-enrichment. Under the framework of Johnson et al. (2017), the formation of such a massive cluster would require a very high star formation rate surface density. Such intense star formation could be triggered by a major merger in M31's early assembly, but there does not seem to be any remaining evidence for such a merger, nor are there other stars or GCs that appear to be currently associated with G1.

Finally, it is worth noting that despite its unusual properties, G1 is not unique. There are several other massive, metal-rich clusters in M31's halo, some of which may be located in the outer halo. Any formation scenario for G1 should also be able to reproduce the properties of these clusters as well.

This analysis has added to the mystery surrounding G1 and the formation of M31's outer halo in general (McConnachie et al. 2018; Mackev et al. 2019). Additional imaging and spectroscopy of nearby field stars can help assess the presence of faint streams surrounding G1, while deeper imaging of the cluster itself could reveal more about possible intracluster abundance spreads. Additional IL spectroscopic observations further in the blue or the infrared could also provide more information about abundance spreads within the cluster. On the theoretical front, 
more detailed modelling of G1's orbit could reveal associations with other GCs or stellar streams. Such information would further help to untangle M31's complex early assembly, the properties of its satellite dwarfs, and the nature of massive clusters like G1.

\section{ACKNOWLEDGMENTS}

The authors thank the referee, Claudia Maraston, for suggestions that have greatly improved this manuscript. The authors especially thank the observing specialists at Apache Point Observatory and McDonald Observatory for their assistance with these observations. The HobbyEberly Telescope (HET) is a joint project of the University of Texas at Austin, the Pennsylvania State University, Ludwig-Maximilians-Universität München, and Georg-August-Universität Göttingen. The HET is named in honor of its principal benefactors, William P. Hobby and Robert E. Eberly. GW acknowledges funding from the Kenilworth Fund of the New York Community Trust. This work has made use of BaSTI web tools. This research has made use of the SIMBAD database, operated at CDS, Strasbourg, France.

\section{DATA AVAILABILITY}

The data underlying this article will be shared on reasonable request to the corresponding author after completion of the final paper in this series.

\section{REFERENCES}

Alpaslan, M. 2009, arXiv:0912.4755

Amarsi, A.M., Lind, K., Asplund, M., Barklem, P.S., \& Collet, R. 2016, MNRAS, 463, 1518

Armandroff, T.E. \& Zinn, R. 1988, AJ, 96, 92

Asplund, M., Grevesse, N., Sauval, J.A., \& Scott, P. 2009,

ARA\&A, 47, 481

Bastian, N., \& Lardo, C. 2018, ARA\&A, 56, 83

Bastian, N., Usher, C., Kamann, S., et al. 2019, MNRAS, 489, L80

Baumgardt, H., Makino, J., Hut, P., et al. 2003, ApJ, 589, L25

Baumueller, D. \& Gehren, T. 1997, A\&A, 325, 1088

Bekki, K. \& Chiba, M. 2004, A\&A, 417, 437

Bellini, A., Bedin, L.R., Piotto, G., Milone, A.P., Marino,

A.F., \& Villanova, S. 2010, AJ, 140, 631

Bisterzo, S., Gallino, R., Pignatari, M., et al. 2004, Memorie della Societa Astronomica Italiana, 75, 741

Böker, T., Sarzi, M., McLaughlin, D. E., et al. 2004, AJ, 127, 105

Brodie, J.P. \& Strader, J. 2006, ARA\&A, 44, 193

Burris, D. L., Pilachowski, C. A., Armandroff, T. E., et al.

2000, ApJ, 544, 302

Caldwell, N., Schiavon, R., Morrison, H., et al. 2011, AJ, 141, 61

Cappellari, M. \& Emsellem, E. 2004, PASP, 116, 138

Carretta, E., Bragaglia, A., Gratton, R., \& Lucatello, S. 2009, A\&A, 505, 139

Carretta, E., Bragaglia, A., Gratton, R. G., et al. 2010, A\&A, 516, A55
Castelli, F. \& Kurucz, R. L. 2004, in IAU Symp. 210, Modelling of Stellar Atmospheres, ed. N. Piskunov, W.W. Weiss, \& D. F. Gray (San Francisco: ASP), A20

Cohen, J. G. 2006, ApJ, 653, L21

Colucci, J.E., Bernstein, R.A., Cameron, S., McWilliam, A., \& Cohen, J.G. 2009, ApJ, 704, 385

Colucci, J.E., Bernstein, R.A., Cameron, S.A., \& McWilliam, A. 2011, ApJ, 735, 55

Colucci, J.E., Bernstein, R.A., Cameron, S.A., \& McWilliam, A. $2012, \mathrm{ApJ}, 746,29$

Colucci, J.E., Bernstein, R.B., \& Cohen, J.G. 2014, ApJ, 797, 116

Colucci, J. E., Bernstein, R. A., \& McWilliam, A. 2017, ApJ, 834, 105

D’Orazi, V., Gratton, R. G., Pancino, E., et al. 2011, A\&A, 534, A29

Fahrion, K., Müller, O., Rejkuba, M., et al. 2020, A\&A, 634, A53

Faria, D., Johnson, R. A., Ferguson, A. M. N., et al. 2007, AJ, 133,1275

Federici, L., Cacciari, C., Bellazzini, M., et al. 2012, A\&A, 544, A155

Ferguson, A. M. N., Irwin, M. J., Ibata, R. A., et al. 2002, AJ, 124, 1452

Ferguson, A. M. N., Johnson, R. A., Faria, D. C., et al. 2005, ApJ, 622, L109

Ferguson, J.W., Alexander, D.R., Allard, F. et al. 2005, ApJ, 623,585

Foster, C., Forbes, D.A., Proctor, R.N., Strader, J., Brodie, J.P., \& Spitler, L.R. 2010, AJ, 139, 1566

Foster, C., Spitler, L.R., Romanowsky, A.J., et al. 2011, MN-

RAS, 415, 3393

Freeman, K.C. \& Rodgers, A.W. 1975, ApJ, 201, L71

Freeman, K. C., \& Norris, J. 1981, ARA\&A, 19, 319

Fuentes-Carrera, I., Jablonka, P., Sarajedini, A., et al. 2008, A\&A, 483, 769

Galleti, S., Bellazzini, M., Buzzoni, A, Federici, L., \& Fusi Pecci, F. 2009, A\&A, 508, 1285

Gebhardt, K., Rich, R. M., \& Ho, L. C. 2002, ApJ, 578, L41

Gebhardt, K., Rich, R. M., \& Ho, L. C. 2005, ApJ, 634, 1093

Georgiev, I. Y., Hilker, M., Puzia, T. H., et al. 2009, MNRAS, 396, 1075

Harris, W. E. 1996, AJ, 112, 1487 (2010 edition)

Harris, W. E., Harris, G. L. H., \& Alessi, M. 2013, ApJ, 772, 82

Helmi, A., Babusiaux, C., Koppelman, H. H., et al. 2018, Nature, 563,85

Hinkle, K., Wallace, L., Livingston, W., Ayres, T., Harmer, D., \& Valenti, J. 2003, in The Future of Cool-Star Astrophysics: 12th Cambridge Workshop on Cool Stars, Stellar Systems, and the Sun (2001 July 30 - August 3), eds. A. Brown, G.M. Harper, and T.R. Ayres, (University of Colorado), 851

Hollyhead, K., Kacharov, N., Lardo, C., et al. 2017, MNRAS, 465, L39

Hollyhead, K., Martocchia, S., Lardo, C., et al. 2019, MNRAS, 484, 4718

Huchra, J. P., Brodie, J. P., \& Kent, S. M. 1991, ApJ, 370, 495

Ibata, R.A., Gilmore, G., \& Irwin, M.J. 1995, MNRAS, 277, 781

Ibata, R. A., Lewis, G. F., McConnachie, A. W., et al. 2014, ApJ, 780, 128

Ibata, R. A., Bellazzini, M., Malhan, K., et al. 2019, Nature Astronomy, 3, 667

Johnson, J.A., Ivans, I.I., \& Stetson, P.B. 2006, ApJ, 640, 801 Johnson, J. A., Ivans, I. I., \& Stetson, P. B. 2006, ApJ, 640, 801 
Johnson, C.I. \& Pilachowski, C.A. 2010, ApJ, 722, 1373

Johnson, C. I., Caldwell, N., Rich, R. M., et al. 2017, ApJ, 836,168

Johnston, K. V., Bullock, J. S., Sharma, S., et al. 2008, ApJ, 689,936

Johnston, E. J., Puzia, T. H., D'Ago, G., et al. 2020, MNRAS, 495, 2247

Kacharov, N., Neumayer, N., Seth, A. C., et al. 2018, MN-

RAS, 480, 1973

Kim, S., Staveley-Smith, L., Dopita, M. A., et al. 1998, ApJ,

503, 674. doi:10.1086/306030

Koch, A. \& McWilliam, A. 2008, AJ, 135, 1551

Kroupa, P. 2002, Science, 295,82

Kurucz, R.L. 2005, Mem. Soc. Astron. Italiana, 8, 189

Larsen, S. S., Brodie, J. P., \& Strader, J. 2017, A\&A, 601, A96

Larsen, S. S., Brodie, J. P., Wasserman, A., et al. 2018a, A\&A, 613, A56

Larsen, S. S., Pugliese, G., \& Brodie, J. P. 2018b, A\&A, 617, A119

Leigh, N., Böker, T., \& Knigge, C. 2012, MNRAS, 424, 2130

Letarte, B., Hill, V., Tolstoy, E., et al. 2010, A\&A, 523, A17

Lind, K., Asplund, M., Barklem, P.S., \& Belyaev, A.K. 2011,

A\&A, 528, A103

Lind, K., Bergemann, M., \& Asplund, M. 2012, MNRAS, 427, 50

Ma, J., de Grijs, R., Chen, D., et al. 2007, MNRAS, 376, 1621 Mackey, A. D., Huxor, A. P., Ferguson, A. M. N., et al. 2010, ApJ, 717, L11

Mackey, A. D., Ferguson, A. M. N., Huxor, A. P., et al. 2019, MNRAS, 484, 1756

Maoz, D., Sharon, K., \& Gal-Yam, A. 2010, ApJ, 722, 1879

Marino, A.F., Milone, A.P., Piotto, G. 2011a, ApJ, 731, 64

Marino, A.F., Sneden, C., Kraft, R.P., et al. 2011b, A\&A, 532, 8

Marino, A.F., Milone, A.P., Piotto, G., et al. 2012, ApJ, 746, 14

Marino, A. F., Milone, A. P., Karakas, A. I., et al. 2015, MNRAS, 450, 815

Marino, A. F., Milone, A. P., Renzini, A., et al. 2019, MN-

RAS, 487, 3815

Massari, D., Mucciarelli, A., Ferraro, F.R., et al. 2014, ApJ, 795, 22

Massari, D., Koppelman, H. H., \& Helmi, A. 2019, A\&A, 630, L4

Matteucci, F. \& Brocato, E. 1990, ApJ, 365, 539

Mayall, N.U. \& Eggen, O.J. 1953, PASP, 65, 24

McConnachie, A. W. 2012, AJ, 144, 4

McConnachie, A. W., Ibata, R., Martin, N., et al. 2018, ApJ, 868,55

McWilliam, A., Preston, G.W., Sneden, C., \& Searle, L. 1995b, AJ, 109, 2757

McWilliam, A. \& Bernstein, R. 2008, ApJ, 684, 326

McWilliam, A., Wallerstein, G., \& Mottini, M. 2013, ApJ, 778, 149

Mészáros, S., Masseron, T., García-Hernández, D. A., et al. 2020, MNRAS, 492, 1641

Meylan, G., Sarajedini, A., Jablonka, P., Djorgovski, S.G., Bridges, T., \& Rich, R.M. 2001, AJ, 122, 830

Miller-Jones, J. C. A., Wrobel, J. M., Sivakoff, G. R., et al. 2012, ApJ, 755, L1

Milone, A. P., Piotto, G., Renzini, A., et al. 2017, MNRAS, 464, 3636

Mucciarelli, A., Carretta, E., Origlia, L., et al. 2008, AJ, 136, 375

Mucciarelli, A., Origlia, L., \& Ferraro, F.R. 2010, ApJ, 717, 277

Mucciarelli, A., Bellazzini, M., Ibata, R., et al. 2017, A\&A, 605, A46
Nardiello, D., Piotto, G., Milone, A. P., et al. 2019, MNRAS, 485,3076

Neumayer, N., Seth, A., \& Böker, T. 2020, A\&ARv, 28, 4

Ordenes-Briceño, Y., Puzia, T. H., Eigenthaler, P., et al. 2018, ApJ, 860, 4

Peng, E. W., Jordán, A., Côté, P., et al. 2006, ApJ, 639, 95

Peñarrubia, J., Zucker, D. B., Irwin, M. J., et al. 2011, ApJ, 727, L2

Perina, S., Bellazzini, M., Buzzoni, A., et al. 2012, A\&A, 546, A31

Pietrinferni, A., Cassisi, S., Salaris, M., et al. 2004, ApJ, 612, 168

Pietrinferni, A., Cassisi, S., Salaris, M., et al. 2006, ApJ, 642, 797

Pignatari, M., Gallino, R., Heil, M., et al. 2010, ApJ, 710, 1557

Ramsey, L.W., Adams, M.T., Barnes, T.G., et al. 1998, Proc. SPIE, 3352, 34

Reddy, B.E., Lambert, D.L., \& Prieto, C.A. 2006, MNRAS, 367, 1329

Reitzel, D.B., Guhathakurta, P., \& Rich, R.M. 2004, AJ, 127, 2133

Rich, R.M., Mighell, K.J., Freedman, W.L., \& Neill, J.D. 1996 AJ, 111, 768

Richardson, J. C., Ferguson, A. M. N., Johnson, R. A., et al. 2008, AJ, 135, 1998

Roederer, I. U. 2011, ApJ, 732, L17

Sakari, C. M., Venn, K. A., Irwin, M., et al. 2011, ApJ, 740, 106

Sakari, C.M., Shetrone, M., Venn, K., McWilliam, A., \& Dotter, A. 2013, MNRAS, 434, 358

Sakari, C.M., Venn, K., Shetrone, M., Dotter, A., \& Mackey, D. 2014, MNRAS, 443, 2285

Sakari, C. 2014, Ph.D. dissertation, University of Victoria

Sakari, C.M., Venn, K.A., Mackey, D., et al. 2015, MNRAS, 448, 1314

Sakari, C.M. \& Wallerstein, G. 2016, MNRAS, 456, 831

Sakari, C.M., Shetrone, M.D., Schiavon, R.P., et al. 2016, ApJ, in press

Sakari, C. M., McWilliam, A., \& Wallerstein, G. 2017, MNRAS, 467, 1112

Sakari, C. M., Placco, V. M., Farrell, E. M., et al. 2018, ApJ, 868,110

Sánchez-Janssen, R., Côté, P., Ferrarese, L., et al. 2019, ApJ, 878,18

Schiavon, R.P., Rose, J.A., Courteau, S., \& MacArthur, L.A. 2004, ApJ, 608, 33

Schiavon, R. P., Caldwell, N., Conroy, C., et al. 2013, ApJ, 776, L7

Shetrone, M., Venn, K. A., Tolstoy, E., et al. 2003, AJ, 125, 684

Shetrone, M.D., Cornell, M.E., Fowler, J.R., et al. 2007, PASP, 119, 556

Sneden, C. 1973, ApJ, 184, 839

Sneden, C., Kraft, R. P., Shetrone, M. D., et al. 1997, AJ, 114, 1964

Spengler, C., Côté, P., Roediger, J., et al. 2017, ApJ, 849, 55

Strader, J., Smith, G. H., Larsen, S., et al. 2009, AJ, 138, 547

Thygesen, A. O., Sbordone, L., Andrievsky, S., et al. 2014, A\&A, 572, A108

Thygesen, A. O., Sbordone, L., Ludwig, H.-G., et al. 2016, A\&A, 588, A66

Tolstoy, E., Hill, V., \& Tosi, M. 2009, ARA\&A, 47, 371

Tull, R.G., 1998, Proc. SPIE, 3355, 387

Usher, C., Forbes, D.A., Brodie, J.P., et al. 2012, MNRAS, 426, 1475

Usher, C., Beckwith, T., Bellstedt, S., et al. 2019, MNRAS, 482,1275 
van den Bergh, S. 1969, ApJS, 19, 145

VandenBerg, D. A., Bergbusch, P. A., Dotter, A., et al. 2012, ApJ, 755, 15

Vasiliev, E. \& Belokurov, V. 2020, MNRAS, 497, 4162

Veljanoski, J., Mackey, A. D., Ferguson, A. M. N., et al. 2014, MNRAS, 442, 2929

Venn, K.A., Irwin, M., Shetrone, M.D., et al. 2004, AJ, 128, 1177

Villanova, S., Geisler, D., Gratton, R.G., \& Cassisi, S. 2014, ApJ, 791, 107

Yong, D., Roederer, I.U., Grundahl, F., et al. 2014, MNRAS, 441, 3396

APPENDIX A: ABUNDANCES FROM INDIVIDUAL SPECTRAL LINES 
Sakari et al.

Table A1. Abundances for individual spectral lines.

\begin{tabular}{|c|c|c|c|c|c|}
\hline$\overline{\text { Element }}$ & $\begin{array}{l}\text { Wavelength } \\
(\AA)\end{array}$ & $\begin{array}{c}\mathrm{EP} \\
(\mathrm{eV})\end{array}$ & $\overline{\log g f}$ & $\begin{array}{c}\text { G1 } \\
\log \epsilon\end{array}$ & $\begin{array}{c}47 \text { Tuc } \\
\log \epsilon\end{array}$ \\
\hline$\overline{\mathrm{Fe} I}$ & 5068.77 & 2.94 & -1.23 & $6.50 \pm 0.20$ & $6.70 \pm 0.10$ \\
\hline $\mathrm{Fe} I$ & 5159.06 & 4.28 & -0.82 & $6.50 \pm 0.20$ & $6.80 \pm 0.10$ \\
\hline $\mathrm{Fe} \mathrm{I}$ & 5191.45 & 3.04 & -0.55 & $6.40 \pm 0.20$ & $6.70 \pm 0.10$ \\
\hline $\mathrm{Fe} \mathrm{I}$ & 5215.18 & 3.26 & -0.86 & $6.35 \pm 0.20$ & $6.70 \pm 0.10$ \\
\hline $\mathrm{Fe} \mathrm{I}$ & 5302.30 & 3.28 & -0.73 & $6.55 \pm 0.20$ & $6.80 \pm 0.10$ \\
\hline $\mathrm{Fe} \mathrm{I}$ & 5364.87 & 4.44 & 0.22 & $6.40 \pm 0.20$ & $6.70 \pm 0.10$ \\
\hline $\mathrm{Fe} \mathrm{I}$ & 5383.37 & 4.31 & 0.50 & $6.60 \pm 0.10$ & $6.70 \pm 0.10$ \\
\hline $\mathrm{Fe} \mathrm{I}$ & 5415.20 & 4.38 & 0.50 & $6.45 \pm 0.10$ & $6.60 \pm 0.10$ \\
\hline $\mathrm{Fe} \mathrm{I}$ & 5501.47 & 0.96 & -3.05 & $6.50 \pm 0.20$ & $6.70 \pm 0.10$ \\
\hline $\mathrm{Fe} \mathrm{I}$ & 5569.62 & 3.41 & -0.52 & $6.55 \pm 0.10$ & $6.70 \pm 0.10$ \\
\hline $\mathrm{Fe} \mathrm{I}$ & 5686.53 & 4.55 & -0.63 & $6.50 \pm 0.20$ & $6.80 \pm 0.10$ \\
\hline $\mathrm{Fe} \mathrm{I}$ & 5976.78 & 3.94 & -1.50 & $6.70 \pm 0.10$ & $6.85 \pm 0.10$ \\
\hline $\mathrm{Fe} \mathrm{I}$ & 6003.01 & 3.88 & -1.10 & $6.30 \pm 0.10$ & $6.60 \pm 0.10$ \\
\hline $\mathrm{Fe} \mathrm{I}$ & 6024.06 & 4.55 & -0.12 & $6.50 \pm 0.10$ & $6.65 \pm 0.10$ \\
\hline $\mathrm{Fe} I$ & 6079.01 & 4.65 & -1.12 & $6.65 \pm 0.15$ & $6.80 \pm 0.05$ \\
\hline $\mathrm{Fe} \mathrm{I}$ & 6137.69 & 2.59 & -1.40 & $6.55 \pm 0.20$ & $6.70 \pm 0.20$ \\
\hline $\mathrm{Fe} \mathrm{I}$ & 6180.20 & 2.73 & -2.78 & $6.60 \pm 0.20$ & $6.70 \pm 0.10$ \\
\hline Fe I & 6219.28 & 2.20 & -2.45 & $6.25 \pm 0.20$ & $6.35 \pm 0.10$ \\
\hline $\mathrm{Fe} \mathrm{I}$ & 6302.49 & 3.68 & -1.13 & $6.40 \pm 0.20$ & $6.70 \pm 0.10$ \\
\hline $\mathrm{Fe} \mathrm{I}$ & 6322.69 & 2.59 & -2.43 & $6.45 \pm 0.20$ & $6.80 \pm 0.10$ \\
\hline $\mathrm{Fe} \mathrm{I}$ & 6380.74 & 4.18 & -1.40 & $6.60 \pm 0.20$ & $6.80 \pm 0.10$ \\
\hline $\mathrm{Fe} \mathrm{I}$ & 6411.65 & 3.65 & -0.59 & $6.10 \pm 0.20$ & $6.50 \pm 0.10$ \\
\hline $\mathrm{Fe} I$ & 6475.62 & 2.56 & -2.94 & $6.90 \pm 0.20$ & $7.20 \pm 0.10$ \\
\hline $\mathrm{Fe} \mathrm{I}$ & 6518.37 & 2.83 & -2.75 & $6.70 \pm 0.20$ & $7.00 \pm 0.10$ \\
\hline $\mathrm{Fe} \mathrm{I}$ & 6533.93 & 4.56 & -1.46 & $6.80 \pm 0.20$ & $6.90 \pm 0.10$ \\
\hline $\mathrm{Fe}$ II & 5534.85 & 3.25 & -2.90 & $6.55 \pm 0.10$ & $6.70 \pm 0.10$ \\
\hline $\mathrm{Fe}$ II & 6456.39 & 3.90 & -2.20 & $6.79 \pm 0.10$ & $6.90 \pm 0.10$ \\
\hline $\mathrm{Na} \mathrm{I}$ & 5682.63 & 2.10 & -0.70 & $5.78 \pm 0.10$ & $5.84 \pm 0.10$ \\
\hline $\mathrm{Na} \mathrm{I}$ & 5688.20 & 2.10 & -0.45 & $5.73 \pm 0.10$ & $5.84 \pm 0.10$ \\
\hline $\mathrm{Na} \mathrm{I}$ & 6154.23 & 2.10 & -1.56 & $6.00 \pm 0.10$ & $5.89 \pm 0.10$ \\
\hline $\mathrm{Na} \mathrm{I}$ & 6160.75 & 2.10 & -1.26 & $5.92 \pm 0.20$ & $5.89 \pm 0.10$ \\
\hline Mg I & 5528.31 & 4.34 & -0.62 & $7.00 \pm 0.10$ & $7.10 \pm 0.10$ \\
\hline Mg I & 5711.09 & 4.34 & -1.83 & $7.00 \pm 0.20$ & $7.40 \pm 0.10$ \\
\hline $\mathrm{Al} \mathrm{I}$ & 6696.02 & 3.14 & -1.35 & $6.14 \pm 0.20$ & $5.95 \pm 0.10$ \\
\hline $\mathrm{Al} \mathrm{I}$ & 6698.67 & 3.14 & -1.65 & $6.24 \pm 0.20$ & $6.05 \pm 0.10$ \\
\hline $\mathrm{Ca} \mathrm{I}$ & 5581.97 & 2.52 & -0.71 & $5.70 \pm 0.10$ & $5.74 \pm 0.10$ \\
\hline Ca I & 5588.75 & 2.52 & 0.21 & $5.80 \pm 0.10$ & $5.94 \pm 0.10$ \\
\hline $\mathrm{Ca} \mathrm{I}$ & 5590.11 & 2.52 & -0.71 & $5.60 \pm 0.10$ & $5.98 \pm 0.10$ \\
\hline $\mathrm{Ca} \mathrm{I}$ & 5601.28 & 2.52 & -0.69 & $5.83 \pm 0.10$ & $5.94 \pm 0.10$ \\
\hline $\mathrm{Ca} \mathrm{I}$ & 5857.45 & 2.93 & 0.23 & $6.00 \pm 0.10$ & $6.04 \pm 0.10$ \\
\hline Ca I & 6166.44 & 2.52 & -0.90 & $5.50 \pm 0.20$ & $5.74 \pm 0.10$ \\
\hline $\mathrm{Ca} \mathrm{I}$ & 6439.07 & 2.52 & 0.47 & $5.53 \pm 0.10$ & - \\
\hline $\mathrm{Ca} \mathrm{I}$ & 6455.60 & 2.52 & -1.35 & $5.75 \pm 0.10$ & $5.84 \pm 0.10$ \\
\hline $\mathrm{Ca} \mathrm{I}$ & 6572.78 & 0.00 & -4.29 & $5.73 \pm 0.15$ & $5.94 \pm 0.10$ \\
\hline Ti I & 4991.07 & 0.84 & 0.45 & $4.24 \pm 0.10$ & $4.25 \pm 0.10$ \\
\hline Ti I & 4999.50 & 0.83 & 0.32 & $4.34 \pm 0.10$ & $4.55 \pm 0.10$ \\
\hline Ti I & 5210.38 & 0.05 & -0.82 & $4.44 \pm 0.10$ & $4.65 \pm 0.10$ \\
\hline Ti I & 6743.12 & 0.90 & -1.63 & $4.24 \pm 0.15$ & $4.45 \pm 0.10$ \\
\hline Ti II & 5336.79 & 1.58 & -1.60 & $4.24 \pm 0.10$ & $4.55 \pm 0.10$ \\
\hline Ti II & 5418.77 & 1.58 & -2.13 & $4.24 \pm 0.10$ & $4.50 \pm 0.10$ \\
\hline Cr I & 5206.02 & 0.94 & 0.02 & $4.53 \pm 0.05$ & $4.84 \pm 0.10$ \\
\hline Cr I & 5345.80 & 1.00 & -0.95 & $4.53 \pm 0.10$ & $4.64 \pm 0.10$ \\
\hline $\mathrm{Mn} \mathrm{I}^{a}$ & 4823.52 & 2.32 & 0.14 & $4.42 \pm 0.10$ & $4.63 \pm 0.15$ \\
\hline $\mathrm{Mn} \mathrm{I}^{a}$ & 5516.77 & 2.18 & -1.85 & $4.32 \pm 0.10$ & $4.43 \pm 0.10$ \\
\hline $\mathrm{Mn} \mathrm{I}^{a}$ & 6013.51 & 3.07 & -0.35 & $4.12 \pm 0.20$ & $4.28 \pm 0.10$ \\
\hline $\mathrm{Ni} \mathrm{I}$ & 6643.63 & 1.68 & -2.22 & $5.21 \pm 0.10$ & $5.37 \pm 0.10$ \\
\hline $\mathrm{Ni} \mathrm{I}$ & 6767.77 & 1.83 & -2.14 & $5.31 \pm 0.10$ & $5.52 \pm 0.10$ \\
\hline $\mathrm{Cu} \mathrm{I}{ }^{a}$ & 5782.13 & 1.64 & -1.72 & $2.58 \pm 0.20$ & $3.29 \pm 0.10$ \\
\hline Zn I & 4810.53 & 4.08 & -0.14 & $3.85 \pm 0.15$ & $3.86 \pm 0.10$ \\
\hline Y II & 5087.42 & 1.08 & -0.17 & $1.20 \pm 0.20$ & $1.46 \pm 0.15$ \\
\hline Y II & 5200.41 & 0.99 & -0.57 & $1.00 \pm 0.20$ & $1.36 \pm 0.10$ \\
\hline $\mathrm{Ba} \mathrm{II}{ }^{a}$ & 5853.67 & 0.60 & -1.01 & $1.17 \pm 0.20$ & $1.38 \pm 0.20$ \\
\hline $\mathrm{Ba} \mathrm{II}{ }^{a}$ & 6141.71 & 0.70 & -0.08 & $1.07 \pm 0.10$ & $1.53 \pm 0.05$ \\
\hline $\mathrm{Ba} \mathrm{II}{ }^{a}$ & 6496.90 & 0.60 & -0.38 & $1.37 \pm 0.20$ & $1.48 \pm 0.10$ \\
\hline $\mathrm{Eu} \mathrm{II}{ }^{a}$ & 6645.06 & 1.38 & 0.20 & $<0.01$ & $0.12 \pm 0.20$ \\
\hline
\end{tabular}

${ }^{a}$ Line has HFS or isotopic splitting. 